\title{
CONLEY INDEX ORIENTATIONS
}

\author{
AXEL JäNIG
}

\begin{abstract}
The homotopy Conley index along heteroclinic solutions of certain parabolic evolution equations is zero under appropriate assumptions. This result implies that the so-called connecting homomorphism associated with a heteroclinic solution is an isomorphism. Hence, using $\mathbb{Z}$-coefficients it can be viewed as either 1 or -1 - depending on the choice of generators for the homology Conley index. We develop a method to choose such generators, and compute the connecting homomorphism relative to these generators.
\end{abstract}

\section{Introduction}

The homotopy Conley index along heteroclinic solutions of certain parabolic evolution equations is zero under appropriate assumptions (see [6]). These assumptions generalize the setting in which the Morse-Smale-Witten chain complex on finite-dimensional manifolds is constructed.

This result implies that the so-called connecting homomorphism associated with a heteroclinic solution is an isomorphism. Hence, using $\mathbb{Z}$-coefficients it can be viewed as either 1 or -1 . To be more precise, suppose we are given a semiflow $\pi$ and a heteroclinic solution $u(t)$ with $u(t) \rightarrow e^{ \pm}$as $t \rightarrow \pm \infty$. We are only interested in the case where $e^{+}$and $e^{-}$are hyperbolic equilibria with adjacent Morse indices, that is, $m\left(e^{+}\right)+1=m\left(e^{-}\right)$. It is well-known that

2010 Mathematics Subject Classification. Primary 37B30; Secondary 37D15.

Key words and phrases. Conley index theory, Morse decompositions, reaction diffusion equations. 
for a hyperbolic equilibrium $e$ with Morse-index $m(e)$, the homology Conley index $\left({ }^{1}\right)$ with coefficients in $\mathbb{Z}$ satisfies

$$
H_{q}\langle\pi,\{e\}\rangle \approx \begin{cases}\mathbb{Z} & \text { if } q=m(e) \\ 0 & \text { if } q \neq m(e)\end{cases}
$$

Of course, considering only one solution, we can choose generators such that the connecting homomorphism $\delta_{q}: H_{q}\left\langle\pi,\left\{e^{-}\right\}\right\rangle \rightarrow H_{q-1}\left\langle\pi,\left\{e^{+}\right\}\right\rangle$is 1 (or -1 ). However, given two heteroclinic solutions $u_{1}(t)$ and $u_{2}(t)$ connecting the same pair of equilibria, it is not clear whether their connecting homomorphisms agree.

Suppose that there exist a stable manifold $W^{s}\left(e^{+}\right)$and an unstable manifold $W^{u}\left(e^{-}\right)$which intersect transversally. Then the signs of connecting homomorphisms can be expressed depending on previously chosen orientations for $T W^{u}\left(e^{+}\right)$and $T W^{u}\left(e^{-}\right)$(see [3], [10]). However, it seems that the aforementioned proofs cover only gradient flows. Note that we also do not require a global orientation.

Our approach is to compute the connecting homomorphism for a given heteroclinic solution independently of the other solutions. The connecting homomorphism is determined by special triples of closed subspaces, the so-called FM-index triples. Suppose we are given FM-index triples $\left(N_{1}, N_{2}, N_{3}\right)$ and $\left(M_{1}, M_{2}, M_{3}\right)$ for two distinct heteroclinic solutions connecting the same pair of equilibria. $\left(N_{1}, N_{2}\right)$ and $\left(M_{1}, M_{3}\right)$ (resp. $\left(N_{2}, N_{3}\right)$ and $\left(M_{2}, M_{3}\right)$ are then both FM-index pairs for the repeller $\left\{e^{-}\right\}$(resp. the attractor $\left\{e^{+}\right\}$).

Recall that the categorial Conley index is a subcategory of the homotopy category of pointed spaces, whose objects are certain FM-index pairs representing the index. We call the unique, designated morphism between to FM-index pairs inner morphism. A more detailed exposition can be found in Section 2 or in [2], which is used as a reference.

$\left(N_{1} / N_{2}, N_{2} / N_{2}\right)$ and $\left(M_{1} / M_{2}, M_{2} / M_{2}\right)$ are objects of the categorial Conley index $\left({ }^{2}\right)$, so there is a unique inner morphism

$$
\begin{aligned}
\alpha:\left(N_{1} / N_{2}, N_{2} / N_{2}\right) & \rightarrow\left(M_{1} / M_{2}, M_{2} / M_{2}\right) \\
\left(\operatorname{resp} . \beta:\left(N_{2} / N_{3}, N_{3} / N_{3}\right)\right. & \left.\rightarrow\left(M_{2} / M_{3}, M_{3} / M_{3}\right)\right)
\end{aligned}
$$

in the homotopy category of pointed spaces.

Let $\delta_{N, q}$ (resp. $\delta_{M, q}$ ) denote the $q$-th connecting homomorphism which is defined by $\left(N_{1}, N_{2}, N_{3}\right)$ (resp. $\left.\left(M_{1}, M_{2}, M_{3}\right)\right)$. Now, the two FM-index triples (resp. the two heteroclinic solutions) determine the same connecting hommomorphism

${ }^{1}$ ) We will follow [2], but one can also assume that $H_{q}\langle\pi, e\rangle=H_{q}\left(N_{1} / N_{2}, N_{2} / N_{2}\right)$, where $\left(N_{1}, N_{2}\right)$ is a strongly admissible FM-index pair for $\{e\}$ relative to $\pi$.

$\left.{ }^{2}\right)$ provided $\operatorname{cl}\left(N_{1} \backslash N_{2}\right)$ and $\operatorname{cl}\left(M_{1} \backslash M_{2}\right)$ are strongly admissible. 
if and only if

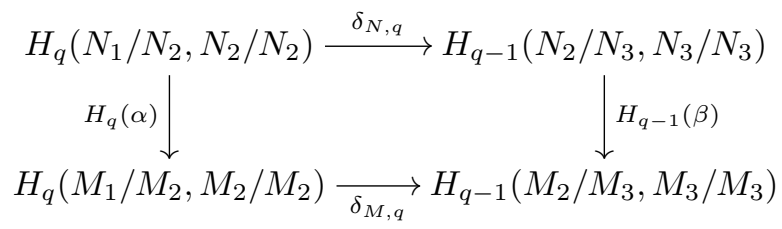

is commutative for all $q \in \mathbb{Z}$. Passing the above diagram to the singular homology of the the categorial Conley index, this means that

$$
H_{q}\left\langle\pi,\left\{e^{\stackrel{\left\langle\delta_{N, q}\right\rangle}{\underset{\left\langle\delta_{M, q}\right\rangle}{H_{q-1}}}}\left\langle\pi,\left\{e^{+}\right\}\right\rangle\right.\right.
$$

is commutative.

We aim to express connecting homomorphisms in terms of integers, that is, relative to a choice of generators. Roughly speaking, typical steps when one computes the homology index of an isolated invariant set are the application of homeomorphisms or continuous changes of the semiflow considered. Unfortunately, it seems that almost every such change - even the smallest one - modifies every index pair representing the categorial Conley index. Therefore, one needs to choose generators in a way that is persistent under (at least) small changes of the semiflow.

In this paper, for $n \in \mathbb{N} \cup\{0\}$ we denote by $D^{n}$ the closed Euclidean unit ball and by $S^{n}$ the Euclidean unit sphere. Notice that $\mathbb{R}^{0}=\{0\} \subset \mathbb{R}, D^{0}:=\{0\}$, and $S^{-1}:=\emptyset$.

Now, let $e$ be a hyperbolic equilibrium, let $n$ denote the Morse index of $e$, and let $f$ be a continuous mapping which maps a neighbourhood of 0 in $\mathbb{R}^{n}$ into a neighbourhood of $e$. It is clear that, given an arbitrary strongly admissible FMindex pair $\left(N_{1}, N_{2}\right)$ for $(\pi,\{e\})$, one has $f(\lambda x) \in N_{1}$ for all $x \in D^{n} \subset \mathbb{R}^{n}$ and all $\lambda \in] 0, \infty$ [ sufficiently small. If it holds additionally that for every $x \in D^{n} \backslash\{0\}$, there exists an $s \in \mathbb{R}^{+}$with $f(\lambda x) \pi s \in N_{2}$, then $f$ is called a seed. Every seed $f$ induces for every strongly admissible FM-index pair $\left(N_{1}, N_{2}\right)$ for $(\pi, e)$ a morphism $\left(D^{n} / S^{n-1},\left[S^{n-1}\right]\right) \rightarrow\left(N_{1} / N_{2},\left[N_{2}\right]\right)$ in the homotopy category of pointed spaces. We will show that these induced morphisms commute with the inner morphisms of the categorial Conley index, that is, they do not depend on the FM-index pair chosen.

Subsequently, we will derive conditions under which a seed induces an isomorphism $\langle f\rangle$ (the Conley index orientation) and conditions under which two seeds induce the same isomorphism. Some of these conditions are rather technical and can be found in Section 3. However, for a large class of parabolic 
evolution equations $\left({ }^{3}\right)$, it turns out that, in some sense, the choice of a basis for the tangential space of the local unstable manifold of $e$ is a seed. Moreover, two such bases induce the same isomorphism if and only if they have the same orientation in the traditional sense (Proposition 4.7). Therefore, we call these isomorphisms orientations.

We define a connected simple system $\mathcal{S}^{n}$, the only object of which is $\left(D^{n} / S^{n-1},\left[S^{n-1}\right]\right)$, and the only morphism is the identity on $D^{n} / S^{n-1}$. Now, we can understand $\langle f\rangle: \mathcal{S}^{n} \rightarrow \mathcal{C}(\pi,\{e\})$ as a morphism of connected simple systems.

There is a natural choice (Definiton 6.14) of ismorphisms $\mu_{q}: \mathbb{Z} \rightarrow H_{q}\left(\mathcal{S}^{q}\right)$, $q \in \mathbb{Z}$, which is unique up to the choice of $\mu_{0}$. Choosing orientations for the attractor and the repeller and using $\mu_{q}$, the connecting homomorphism will be expressed as the multiplication by a number $\theta \in \mathbb{Z}$. Of course, $\theta$ could be defined using an arbitrary family of isomorphisms $\mu_{q}$, but then our formulas for the connecting homomorphism would depend on the Morse indices of the equilibria.

So far, we have sketched the first part of this paper. In Section 6 and 7, we will apply these seed induced orientations to the problem of computing the sign of connecting homomorphisms. We will start with classes of linear skew product semiflows and relate the number $\theta$ introduced above to the linear skew product semiflow. These are exactly the linear skew product semiflows considered in [6]. They are typically $\left({ }^{4}\right)$ defined by equations of the following kind:

$$
\begin{aligned}
\dot{x} & =1-x^{2}, \\
\dot{y}+A y & =F(x) y .
\end{aligned}
$$

The first equation is an ordinary differential equation on the real interval ] $-2,2$ [, $A$ is a sectorial operator satisfying certain assumptions on its spectrum, and $F$ maps the interval $[-2,2]$ continuously (or sufficiently continuously) into $\mathcal{L}\left(X^{\alpha}, X\right)$, where $X$ is a Banach space and $X^{\alpha}$ the $\alpha$-th fractional power space defined with respect to $A$ in the sense of [5]. $(u(t), v(t))$ is a solution of the equation above (resp. its associated semiflow) if $u(t)$ is a solution of $\dot{x}=1-x^{2}$ and $v(t)$ is a mild solution of $\dot{y}+A y=F(u(t)) y$.

We will make some additional assumptions so that there are finitely many eigenvectors of $A-F( \pm 1)$ which belong to the eigenvalues with positive real part. One is then able to show that a family $F_{\lambda}$ for which these eigenvectors (precisely the subspace of $X$ they span) are independent of $\lambda$ induces a family of linear skew product semiflows for which $\theta$, as introduced above, is constant. Hence, one can compute $\theta$ for the simplest case and extend the result to less restrictive assumptions on $F$.

$\left({ }^{3}\right)$ This is the prototypical example; the result is formulated in a more general form.

(4) There is one technical generalization. 
In [6], we related every heteroclinic solution to a linear skew product semiflow. In this paper, we proceed backwards and generalize the technical result for linear skew product semiflows to a formula for the connecting homomorphism for the heteroclinic solution.

We will now demonstrate how the abstract results above can be applied to reaction diffusion equations. Let $\Omega \subset \mathbb{R}^{N}$ be a bounded domain and let $\partial \Omega$ be of class $C^{2}$. Let $2 \leq p<\infty$ and $f: \Omega \times \mathbb{R} \rightarrow \mathbb{R}$. Suppose that for almost all $x \in \Omega$, there is a partial derivative $f_{u}(x, u)$ which is continuous in $u$ and that ess $\sup _{x \in \Omega} \sup _{|u| \leq r}\left|f_{u}(x, u)\right|<\infty$ for all $r \in \mathbb{R}^{+}$. Assume further that $f$ and $(x, u) \mapsto f_{u}(x, u)$ are Carathéodory functions.

We consider the problem:

$$
\begin{aligned}
& u_{t}(x, t)=\Delta u(x, t)+f(x, u(x, t)), \quad t>0, x \in \Omega, \\
& u(x, t)=0, \quad t>0, x \in \partial \Omega \text {. }
\end{aligned}
$$

Let $A_{p}$ denote the closure of $-\Delta:\left\{u \in C^{2}(\Omega): u_{\mid \partial \Omega}=0\right\} \rightarrow L^{p}(\Omega)=: X$ in $W^{2, p}(\Omega)$ and define the Nemitskiu (superposition) operator $\widehat{f} \in C^{1}\left(C(\bar{\Omega}), L^{p}(\Omega)\right.$ ) by

$$
(\widehat{f}(u))(x):=f(x, u(x)) \quad x \in \Omega
$$

so that $(D \widehat{f}(\xi) \eta)(x)=f_{u}(x, \xi(x)) \eta(x)$ almost everywhere.

For $k$ sufficiently large, $A_{p}+k I$ is a positive sectorial operator having compact resolvent. Letting $\xi \in X^{\alpha}$, it follows that all eigenvalues of $A-D \widehat{f}(\xi)$ are real.

Let $p \geq \max \{2, N\}, A:=A_{p}$, and $v: \mathbb{R} \rightarrow X^{\alpha}$ be a heteroclinic mild solution of

$$
\dot{x}+A x=\widehat{f}(x)
$$

and suppose that $v(t) \rightarrow e^{ \pm}$as $t \rightarrow \pm \infty$ in $X^{\alpha}$ (resp. $C(\bar{\Omega})$ ). It follows that $v \in C^{1}\left(\mathbb{R}, L^{p}(\Omega)\right)$. Choosing $0<\alpha<1$ large enough, we can further assume that there is a continuous inclusion $X^{\alpha} \subset C(\bar{\Omega})$ (see [5, Theorem 1.6.1]).

THEOREM $1.1([6])$. Let $u$ be a heteroclinic mild solution of $(1.1)$ with $u(t) \rightarrow$ $e^{ \pm}$as $t \rightarrow \infty$ in $X^{\alpha}$ (resp. $C(\bar{\Omega})$ or $\left.L^{p}(\bar{\Omega})\right)$ and suppose that

(a) $e^{+}, e^{-}$are hyperbolic equilibria,

(b) the Morse indices satisfy $m\left(e^{-}\right)=m\left(e^{+}\right)+1$,

(c) all eigenvalues of $A-D f\left(e^{ \pm}\right)$are simple,

(d) $e^{\lambda t}\left(u(t)-e^{+}\right) \not \rightarrow 0$ for some $\lambda \in \mathbb{R}$, and

(e) every full bounded in $X^{\alpha}$ (resp. $C(\bar{\Omega})$ or $\left.L^{p}(\bar{\Omega})\right)$ mild solution of

$$
\dot{y}+A y=D \widehat{f}(u(t)) y
$$

is a multiple of $\dot{u}$. 
Then the homotopy Conley index $h(\pi, \bar{u})$ of $\bar{u}:=\operatorname{cl}\{u(t): t \in \mathbb{R}\}$ is well-defined and trivial, that is, $h(\pi, \bar{u})=\overline{0}$, where $\pi$ denotes the semiflow which is induced by mild solutions of (1.1).

Suppose that for every $r \in \mathbb{R}$ there exist constants $\delta>0$ and $C \in \mathbb{R}^{+}$such that

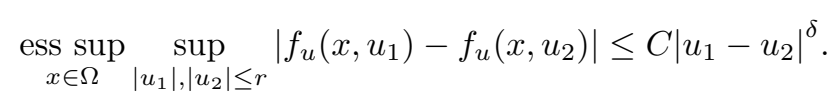

Then for every $1 \leq q \leq \infty, D \widehat{f}: C(\bar{\Omega}) \rightarrow \mathcal{L}\left(L^{q}(\Omega), L^{q}(\Omega)\right)$ is locally Hölder continuous, assumption (d) in Theorem 1.1 holds.

Suppose that $u$ is a solution of $\pi$ for which the assumptions of Theorem 1.1 hold. For each of the equilibria $e^{-}$and $e^{+}$, there are $A-D f\left(e^{-}\right)$-invariant (resp. $\left.A-D f\left(e^{+}\right)\right)$subspaces $E^{-}\left(e^{-}\right)\left(\right.$resp. $\left.E^{-}\left(e^{+}\right)\right)$associated with $\{\operatorname{Re} \sigma(A-$ $\left.\left.D f\left(e^{-}\right)\right)<0\right\}$ (resp. $\left.\left\{\operatorname{Re} \sigma\left(A-D f\left(e^{+}\right)\right)<0\right\}\right)$.

By $E=E_{1} \oplus E_{2}$, we mean that $E_{1}$ and $E_{2}$ are closed linear subspaces of a normed space $E$ with $E_{1} \cap E_{2}=\{0\}$ and $E=E_{1}+E_{2}$. The canonical projection $P: E_{1} \oplus E_{2} \rightarrow E_{1}$ is given by $P\left(e_{1} \oplus e_{2}\right):=e_{1}$.

Provided that the assumptions of Theorem 1.1 hold, we obtain that

$$
\operatorname{dim} E^{-}\left(e^{-}\right)=\operatorname{dim} E^{-}\left(e^{+}\right)+1=: n+1 \quad \text { for some } n \in \mathbb{N} .
$$

Let $\left\{x_{1}, \ldots, x_{n+1}\right\}$ be a basis for $E^{-}\left(e^{-}\right)$consisting of eigenvectors of $A-D f\left(e^{-}\right)$ and let $\left\{y_{1}, \ldots y_{n}\right\}$ denote an (arbitrary) basis for $E^{-}\left(e^{+}\right)$. These bases define toplinear isomorphisms $\Phi_{-1}: \mathbb{R}^{n+1} \rightarrow E^{-}\left(e^{-}\right), \widehat{\Phi}_{-1}: \mathbb{R}^{n} \rightarrow \operatorname{span}\left\{x_{1}, \ldots, x_{i-1}\right.$, $\left.x_{i+1}, \ldots, x_{n+1}\right\}$, and $\Phi_{1}: \mathbb{R}^{n} \rightarrow E^{-}\left(e^{+}\right)$, where we set

$$
\begin{aligned}
\Phi_{-1}\left(\widetilde{\mu}_{1}, \ldots, \widetilde{\mu}_{n+1}\right) & :=\sum_{k=1}^{n+1} \widetilde{\mu}_{k} x_{k}, \\
\widehat{\Phi}\left(\widetilde{\mu}_{1}, \ldots, \widetilde{\mu}_{n}\right) & :=\Phi_{-1}\left(\widetilde{\mu}_{1}, \ldots, \widetilde{\mu}_{i-1}, \widetilde{\mu}_{i+1}, \ldots, \widetilde{\mu}_{n+1}\right), \\
\Phi_{1}\left(\widetilde{\mu}_{1}, \ldots, \widetilde{\mu}_{n}\right) & :=\sum_{k=1}^{n} \widetilde{\mu}_{k} y_{k} .
\end{aligned}
$$

Now, $o_{-1}(\widetilde{\mu}):=e^{-}+\Phi_{-1}(\widetilde{\mu})\left(\right.$ resp. $o_{1}(\widetilde{\mu}):=e^{+}+\Phi_{1}(\widetilde{\mu})$ ) defines a seed for $\left(\pi, e^{-}\right)$(resp. $\left.\left(\pi, e^{+}\right)\right)$. Both seeds induce orientations, that is, they induce isomorphisms of connected simple systems, $\left\langle o_{-1}\right\rangle: \mathcal{S}^{n+1} \rightarrow \mathcal{C}\left(\pi,\left\{e^{-}\right\}\right)$(resp. $\left.\left\langle o_{1}\right\rangle: \mathcal{S}^{n} \rightarrow \mathcal{C}\left(\pi,\left\{e^{+}\right\}\right)\right)$.

Under the assumptions of Theorem 1.1, it holds that $\left\|u(t)-e^{-}\right\|_{\alpha}^{-1}\left(u(t)-e^{-}\right)$ converges to an eigenvector $\pm x_{i}$ of $A-D f\left(e^{-}\right)$as $t \rightarrow-\infty$.

We can further assume that there is an eigenvector $\eta$ of $A-D f\left(e^{+}\right)$with $\left\|u(t)-e^{+}\right\|_{\alpha}^{-1}\left(u(t)-e^{+}\right) \rightarrow \eta$ as $t \rightarrow \infty$. $\eta$ belongs to an eigenvalue $\lambda>0$. If $F$ is an $A-D f\left(e^{+}\right)$invariant subspace of $X$ such that $X=E^{-}\left(e^{+}\right) \oplus \operatorname{span}\{\eta\} \oplus F$, then, for large $t \in \mathbb{R}$, there is a decomposition of $X$, which defines a family $P(t): E^{-}\left(e^{+}\right) \oplus \operatorname{span}\{\dot{u}(t)\} \oplus F \rightarrow E^{-}\left(e^{+}\right)$of canonical projections. Furthermore, 
let $\Pi_{t}$ denote the semigroup associated with the semiflow $\pi$, that is, $\Pi_{t}(x)=x \pi t$, $t \in \mathbb{R}^{+}$. It follows from our assumptions that, for every $t \in \mathbb{R}^{+}, \Pi_{t}$ is continuously differentiable.

We now consider a linear operator $\mathbb{R}^{n} \rightarrow \mathbb{R}^{n}$ :

$$
C(t, \Delta, u):=\Phi_{1}^{-1} \circ P(t+\Delta) \circ D \Pi_{\Delta}(u(t)) \circ \widehat{\Phi} .
$$

It describes the geometrical connection from $E^{-}\left(e^{-}\right)$to $E^{-}\left(e^{+}\right)$given by linearization of $\pi$ along $u$. Let $\delta(u):=\lim _{(t, t+\Delta) \rightarrow(-\infty, \infty)} \operatorname{sgn} \operatorname{det} C(t, \Delta, u)$.

TheOREM 1.2. Suppose that $e^{-}$and $e^{+}$are hyperbolic equilibria. Then for every heteroclinic solution $u(t)$ which satisfies:

(a) $u(t) \rightarrow e^{ \pm}$as $t \rightarrow \pm \infty$,

(b) $\left\|u(t)-e^{-}\right\|_{\alpha}^{-1}\left(u(t)-e^{-}\right) \rightarrow \nu x_{i}$ as $t \rightarrow-\infty, \nu \in\{-1,1\}$, and

(c) the assumptions of Theorem 1.1,

it holds that $\delta(u)$ is well defined and

$$
\partial_{q} \circ H_{q}\left\langle o_{-1}\right\rangle \circ \mu_{q}=\nu \cdot(-1)^{1+i} \cdot \delta(u) \cdot H_{q-1}\left\langle o_{1}\right\rangle \circ \mu_{q-1} .
$$

Here, $\partial_{q}: H_{q}\left\langle\pi,\left\{e^{-}\right\}\right\rangle \rightarrow H_{q-1}\left\langle\pi,\left\{e^{+}\right\}\right\rangle$denotes the $q$-th connecting homomorphism associated with $u$, which is the connecting homomorphism associated with $\left(u(\mathbb{R}) \cup\left\{e^{-}, e^{+}\right\},\left\{e^{+}\right\},\left\{e^{-}\right\}\right)$.

The theorem is an immediate consequence of Theorem 7.4.

\section{Preliminaries}

2.1. Notation. Although most of the notation is more or less standard, a couple of symbols should at least be mentioned. $\mathbb{R}^{+}$(resp. $\mathbb{R}^{-}$) denotes the set of all non-negative (resp. non-negative) real numbers. $W^{u}$ and $W^{s}$ denote unstable respectively stable manifolds, the precise meaning is given when they are used. $\sigma$ is used to designate the spectrum of an operator. The open (resp. closed) ball with radius $r$ and center $x$ is denoted by $B_{r}(x)$ (resp. $B_{r}[x]$ ). If $X$ is a set, then $\# X$ denotes the cardinality of $X$.

Given normed spaces $X$ and $Y$, and a continuous linear operator $F \in$ $\mathcal{L}(X, Y),\|F\|_{X, Y}$ is used sometimes to make the norm unambiguous. $\operatorname{ISO}(X, Y)$ denotes the set of all $F \in \mathcal{L}(X, Y)$ which are toplinear isomorphisms. The notion of fractional power spaces follows [5]. If $F \in \mathcal{L}\left(X^{\alpha}, X^{\beta}\right)$, then $\|F\|_{\alpha, \beta}$ denotes the operator norm.

Finally, if $X, Y$ are topological spaces, $f: X \rightarrow Y$ is a homeomorphism, and $\pi$ is a (local) semiflow on $X$, then $f[\pi]$ is the semiflow on $Y$ which is obtained by conjugacy, that is, $u$ is a solution of $\pi$ if and only if $f \circ u$ is a solution of $f[\pi]$. 
2.2. Conley index. The purpose of this section is to give a short overview over the most important concepts of Conley index theory for semiflows on metric spaces. A more detailed exposition can be found in [2] and [9].

Let $B$ be a topological space and $A \subset B$. Let $(\widetilde{A}, \widetilde{B}):=(A, B)$ if $B \neq \emptyset$ and $(\widetilde{A}, \widetilde{B}):=(A \cup\{*\},\{*\})\left(^{5}\right)$ (endowed with the sum topology) otherwise. Now let $A / B$ denote the set of equivalence classes in $\widetilde{A}$ where $a, \widetilde{a} \in \widetilde{A}$ are related if they are equal or $\{a, \widetilde{a}\} \subset \widetilde{B} . A / B$ is equipped with the quotient topology.

Let $\pi$ be a local semiflow defined on a metric space $X$. A subset $S \subset X$ is called invariant if for every $x \in S$ there exists a full solution $u: \mathbb{R} \rightarrow S$ of $\pi$ through $x$ that is, $u(0)=x$.

Let $Y \subset X,\left(x_{n}\right)_{n}$ a sequence in $Y$, and $\left(t_{n}\right)_{n}$ a sequence in $\mathbb{R}^{+}$such that $t_{n} \rightarrow \infty$ and $x_{n} \pi\left[0, t_{n}\right] \subset Y . Y$ is called $\pi$-admissible if the sequence of endpoints $x_{n} \pi t_{n}$ is relatively compact for every such pair of sequences. We say that $\pi$ does not explode in $Y$ if for every $x \in Y$ either $x \pi t$ is defined for all $t \in \mathbb{R}^{+}$or there is a $t_{0} \in \mathbb{R}^{+}$such that $x \pi\left[0, t_{0}\right]$ is defined and $x \pi t_{0} \notin Y$. $Y$ is called strongly $\pi$-admissible if it is $\pi$-admissible and $\pi$ does not explode in $Y$.

Now let $Z, Y \subset X . Z$ is called $Y$-positively invariant if it holds that $x \pi[0, t] \subset$ $Z$ whenever $x \in Z, x \pi[0, t]$ is defined and $x \pi[0, t] \subset Y$.

$Z$ is called an exit ramp for $Y$ if for every $x \in Y$ with $x \pi[0, t]$ defined and $\not \subset Y$, there is a $t_{0} \in[0, t]$ such that $x \pi\left[0, t_{0}\right] \subset Y$ and $x \pi t_{0} \in Z$.

Definition 2.1 (Definition 2.4 in [2]). A pair $\left(N_{1}, N_{2}\right)$ is called an FM-index pair for $(\pi, S)$ if:

(a) $N_{1}$ and $N_{2}$ are closed subsets of $X$ with $N_{2} \subset N_{1}$ and $N_{2}$ is $N_{1}$-positively invariant;

(b) $N_{2}$ is an exit ramp for $N_{1}$;

(c) $S$ is closed, $S \subset \operatorname{int}_{X}\left(N_{1} \backslash N_{2}\right)$ and $S$ is the largest invariant set in $\operatorname{cl}_{X}\left(N_{1} \backslash N_{2}\right)$.

Assume that there exists a strongly $\pi$-admissible isolating neighbourhood $N$ for $S$, that is, $N \subset X$ is a closed and strongly $\pi$-admissible neighbourhood of $S$ such that $S$ is the largest invariant set in $N$. Then the homotopy Conley index $h(\pi, S)$ is defined to be the homotopy type of $\left(N_{1} / N_{2},\left\{\left[N_{2}\right]\right\}\right)$ where $\left(N_{1}, N_{2}\right)$ is an FM-index pair for $(\pi, S)$ such that $\operatorname{cl}_{X}\left(N_{1} \backslash N_{2}\right)$ is strongly $\pi$-admissible.

Let $u(t)$ satisfy the assumptions of Theorem 1.1 and let $\pi$ denote the semiflow on $X^{\alpha}$ induced by mild solutions of (1.1). Then $S:=\bar{u}:=\operatorname{cl}_{X}\{u(t): t \in \mathbb{R}\}$ is an isolated invariant set admitting a strongly $\pi$-admissible isolating neighbourhood. In particular, the homotopy Conley index $h(\pi, \bar{u})$ is well-defined under these assumptions.

$\left({ }^{5}\right)$ We assume that $* \notin A$. 
Furthermore, $\left(\pi, \bar{u}, e^{+}, e^{-}\right)$is an attractor-repeller decomposition of $\bar{u}$. Suppose we are given an arbitrary attractor-repeller decomposition $\left(\pi, S, A, A^{*}\right)$. A triple $\left(N_{1}, N_{2}, N_{3}\right)$ is an FM-index triple for $\left(\pi, \bar{u}, A, A^{*}\right)$ if $\left(N_{1}, N_{3}\right)$ is an FMindex pair for $(\pi, \bar{u})$ and if $\left(N_{2}, N_{2}\right)$ is an FM-index pair for $e^{+}$. As a consequence, the sequence

$$
\Delta\left(N_{2} / N_{3}\right) / \Delta\left\{\left[N_{3}\right]\right\} \stackrel{i}{\longrightarrow} \Delta\left(N_{1} / N_{3}\right) / \Delta\left\{\left[N_{3}\right]\right\} \stackrel{p}{\longrightarrow} \Delta\left(N_{1} / N_{2}\right) / \Delta\left\{\left[N_{2}\right]\right\}
$$

is weakly exact. Here, $\Delta$ denotes the singular chain functor, which passes a topological space to its singular chain complex. Generally, a sequence of chain maps

$$
C_{1} \stackrel{i}{\longrightarrow} C_{2} \stackrel{p}{\longrightarrow} C_{3}
$$

is called weakly exact if $p \circ i=0$, $\operatorname{ker} i=0$, and $[x] \mapsto p(x)$ induces an isomorphism $H_{q}\left(C_{2} / \operatorname{im} i\right) \rightarrow H_{q}\left(C_{3}\right)$. There is a covariant functor which passes weakly exact sequences of chain maps to long exact sequences in singular homology. Applying this functor to (2.1), one obtains a long exact sequence

$$
\longrightarrow H_{q+1}\left(N_{1} / N_{2},\left\{\left[N_{2}\right]\right\}\right) \stackrel{\partial_{q+1}}{\longrightarrow} H_{q}\left(N_{2} / N_{3},\left\{\left[N_{3}\right]\right\}\right) \longrightarrow H_{q}\left(N_{1} / N_{3},\left\{\left[N_{3}\right]\right\}\right) \longrightarrow
$$

Since these sequences are rather lengthy, we will abbreviate them sometimes by

$$
\longrightarrow H_{q+1}\left[N_{1} / N_{2}\right] \stackrel{\partial_{q+1}}{\longrightarrow} H_{q}\left[N_{2} / N_{3}\right] \longrightarrow H_{q}\left[N_{1} / N_{3}\right] \longrightarrow
$$

The boundary operator $\left(\partial_{q}\right)_{q \in \mathbb{Z}}$ is called the connecting homomorphism associated with the weakly exact sequence or, if appropriate, the attractor-repeller decomposition. In the context of a heteroclinic solution $u$, the connecting homomorphism associated with $u$ will denote the connecting homomorphism of $\bar{u}$.

We will frequently use the notion of $\mathcal{S}$-continuity. It has been defined in $[9$, Definition I.12.1]. Let $\Lambda$ be a metric space and $\left(\pi_{\lambda}, K_{\lambda}\right)_{\lambda \in \Lambda}$ be a family for which the following holds:

(1) For every $\lambda \in \Lambda, \pi_{\lambda}$ is a local semiflow on $X$ and $K_{\lambda} \subset X$.

(2) For every $\lambda \in \Lambda$, there is a strongly $\pi_{\lambda}$-admissible isolating neighbourhood $N_{\lambda}$ for $K_{\lambda}$ relative to $\pi_{\lambda}$.

(3) Whenever $\lambda_{n} \rightarrow \lambda$ in $\Lambda$, then $\pi_{\lambda_{n}} \rightarrow \pi_{\lambda}, N_{\lambda}$ is a strongly $\pi_{\lambda_{n}}$-admissible isolating neighbourhood for $K_{\lambda_{n}}$ relative to $\pi_{\lambda_{n}}$ for all $n$ sufficiently large, and $N_{\lambda}$ is $\left(\pi_{\lambda_{n}}\right)_{n}$-admissible.

These conditions are equivalent to the original definition.

2.3. Categories of connected simple systems. For the convenience of the reader, we will recall a few concepts from [2]. A connected simple system is a small category such that, given any two objects, there is exactly one morphism between them. 
Now, let $\mathcal{K}$ be an arbitrary category, and define another category $[\mathcal{K}]$. The objects of $[\mathcal{K}]$ are all subcategories of $\mathcal{K}$ which are connected simple systems. Let $L$ be an object of $[\mathcal{K}]$. In this context, a morphism of $L$ will be called an inner morphism. A morphism between $\mathcal{K}_{1}$ and $\mathcal{K}_{2}$ in $[\mathcal{K}]$ is a family

$$
\left(f_{A, B}\right)_{A \in \operatorname{Obj}\left(\mathcal{K}_{1}\right), B \in \operatorname{Obj}\left(\mathcal{K}_{2}\right)}
$$

of morphisms in $\mathcal{K}$ such that

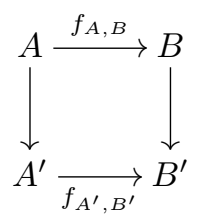

is commutative where the vertical arrows denote the (unique) inner morphisms in $\mathcal{K}_{1}$ respectively $\mathcal{K}_{2}$ (here, we do not follow [2] exactly).

Let $\mathcal{K}_{1}$ and $\mathcal{K}_{2}$ be objects of $[\mathcal{K}], A$ (resp. $B$ ) be an object of $\mathcal{K}_{1}$ (resp. $\mathcal{K}_{2}$ ) and $f$ be a morphism between $A$ and $B$. Then there is exactly one morphism $F$ of $\mathcal{K}$ with $f=F(A, B)$; this morphism is denoted by $[f]$.

Let $\mathcal{T O P}$ denote the category of pointed topological spaces and $\mathcal{H} \mathcal{T}$ the corresponding homotopy category, that is, morphisms of $\mathcal{H} \mathcal{T}$ are equivalence classes of morphisms in $\mathcal{T O P}$, which are continuous, base-point preserving mappings.

As shown in [2], there is a singular homology functor on $[\mathcal{H} \mathcal{T}]$. The $q$-th singular homology is denoted by $\widehat{H}_{q}$ or $H_{q}$ for short.

2.4. Conley indices as a category. Recall that in [2] the categorial Conley-Morse index is defined as a connected simple system, the objects of which are certain $F M$-index pairs of an invariant set admitting a strongly admissible isolating neighbourhood.

Let $(X, d)$ be a metric space, $\pi$ a (local) semiflow on $X$, and $S$ an isolating invariant set admitting a strongly $\pi$-admissible isolating neighbourhood. Then there is an FM-index pair $\left(N_{1}, N_{2}\right)$ for $(\pi, S)$ with the additional property that $\operatorname{cl}\left(N_{1} \backslash N_{2}\right)$ is strongly $\pi$-admissible. In this case, we say that $\left(N_{1}, N_{2}\right)$ is a strongly $\pi$-admissible isolating neighbourhood for $(\pi, K)$. Note that, in general, we neither need nor make the stronger assumption that $N_{1}$ is strongly $\pi$-admissible.

Now, the Conley index $\mathcal{C}(\pi, S)$ of $(\pi, S)$ is an object of $[\mathcal{H} \mathcal{T}]$ (see [2]). The objects of $\mathcal{C}(\pi, S)$ are all pointed spaces of the form $\left(N_{1} / N_{2},\left\{\left[N_{2}\right]\right\}\right)$ where $\left(N_{1}, N_{2}\right)$ is a strongly admissible FM-index pair for $(\pi, S)$. If $\left(N_{1}, N_{2}\right) \subset\left(M_{1}, M_{2}\right)$ are strongly admissible FM-index pairs for $(\pi, S)$, then the inclusion induced (see [9]) morphism $\left(N_{1} / N_{2},\left\{\left[N_{2}\right]\right\}\right) \rightarrow\left(M_{1} / M_{2},\left\{\left[M_{2}\right]\right\}\right)$ is a morphism of $\mathcal{C}(\pi, S)$.

We will use $H_{q}\langle\pi, S\rangle:=\widehat{H}_{q}(\mathcal{C}(\pi, S))$ to denote the homology Conley index of $(\pi, S)$ as defined in [2, Definition 4.3]. The notation of $\pi$ is sometimes omitted. 
Let $(\widetilde{X}, \widetilde{d})$ be another metric space, $\widetilde{\pi}$ a local semiflow on $X$, and $\widetilde{S}$ be an isolating invariant set admitting a strongly $\widetilde{\pi}$-admissible isolating neighbourhood. Then, given a morphism

$$
[f]: \mathcal{C}(\pi, S) \rightarrow \mathcal{C}(\widetilde{\pi}, \widetilde{\mathcal{S}})
$$

there is a unique induced morphism

$$
H_{q}\langle f\rangle:=H_{q}\langle[f]\rangle: H_{q}\langle\pi, S\rangle \rightarrow H_{q}\langle\widetilde{\pi}, \widetilde{S}\rangle .
$$

2.5. Linearizable semiflows. Let $X$ be a Banach space and let $\pi^{\prime}$ be a global semiflow on $X$ generating a $C_{0}$-semigroup of linear operators, that is, for every $t \in \mathbb{R}^{+}$the map $T(t): X \rightarrow X, T(t):=x \pi^{\prime} t$, is linear. We will call such a semiflow linear.

Suppose there is a direct sum $X=X_{1} \oplus X_{2}$ of invariant subspaces, $X_{1}$ is finite-dimensional, $T(t)$ can be uniquely extended to $t \in \mathbb{R}^{-}$to form a $C_{0}$-group on $X_{1}$, and there are constants $M, \delta \in \mathbb{R}^{+} \backslash\{0\}$ such that

$$
\begin{aligned}
& \|T(t) x\| \leq M e^{\delta t}\|x\|, \quad x \in X_{1}, t \in \mathbb{R}^{-}, \\
& \|T(t) x\| \leq M e^{-\delta t}\|x\|, \quad x \in X_{2}, t \in \mathbb{R}^{+} .
\end{aligned}
$$

These are the assumptions of [9, Theorem I.11.1]. Letting $V^{+}(x)$ and $V^{-}(x)$ be defined as in the proof of this theorem, there exists a $\rho \in \mathbb{R}^{+}$such that $N_{1}:=\left\{x \in X: V^{+}(x) \leq \rho\right.$ and $\left.V^{-}(x) \leq \rho\right\}$ and $N_{2}:=\left\{x \in N_{1}: V^{+}(x)=\rho\right\}$ defines a strongly $\pi^{\prime}$-admissible FM-index pair $\left(N_{1}, N_{2}\right)$.

Suppose that $\mathcal{U} \subset X^{\alpha}$ is an open neighbourhood of $0, \pi$ a semiflow on $\mathcal{U}$, and $\{0\}$ an isolated invariant set relative to $\pi$ admitting a strongly $\pi$-admissible isolating neighbourhood.

Definition 2.2. Let $P:=P_{\pi}: X \rightarrow X_{1}$ denote the projection with ker $P=$ $X_{2} . \pi$ is called strongly linearizable (at 0 ) if there exists an $\mathcal{S}$-continuous family $\left(\pi_{\lambda},\{0\}\right)_{\lambda \in[0,1]}$ such that:

(a) $\pi_{1}=\pi$ and

(b) $\pi_{0}$ is a linear semiflow for which the assumptions above hold;

(c) for every $\lambda \in[0,1]$, there exists a neighbourhood $U=U_{\lambda}$ of 0 such that $\left\|x_{n}\right\|^{-1} P x_{n} \rightarrow 0$ whenever $x_{n} \in \operatorname{Inv}_{\pi_{\lambda}}^{+}(U) \backslash\{0\}$ is a sequence with $x_{n} \rightarrow 0$ as $n \rightarrow \infty$.

$\pi^{\prime}:=\pi_{0}$ is called a linearization of $\pi$.

Roughly speaking, the above notion of being strongly linearizable holds for hyperbolic equlibria of the parabolic evolution equations considered in this paper.

Proposition 2.3. Suppose that the semiflow $\pi$ on $\mathcal{U} \subset X^{\alpha}$ is given by mild solutions of a semilinear parabolic equation $\dot{x}+A x=f(x)$ such that:

(a) $A$ is sectorial and has compact resolvent; 
(b) $f: \mathcal{U} \rightarrow X$ is locally Lipschitz continuous; $f(0)=0, f$ has a Fréchet derivative $D f(0)$ at 0 ;

(c) $L:=A-D f(0)$ is hyperbolic.

Then $\pi$ is strongly linearizable.

Proof. For $\lambda \in[0,1]$, let $f_{\lambda}(x):=(1-\lambda)(f(x)-D f(0) x)$ and $\pi_{\lambda}$ be the semiflow defined by mild solutions of $\dot{x}+L x=f_{\lambda}(x)$. Note that $\pi_{1}=\pi$ and $f_{0} \equiv 0$.

Then, $\left(\pi_{\lambda},\{0\}\right)_{\lambda \in[0,1]}$ is an $\mathcal{S}$-continuous family [9, Theorem II.3.5]. As before, let $X=X_{1} \oplus X_{2}$, where $X_{1}$ belongs to $\{\Re \sigma(l)<0\}$ and $X_{2}$ to $\{\Re \sigma(L)>0\}$. This decomposition of $X$ is the same for all $\lambda \in[0,1]$ since $D f_{\lambda}(0)=0$ for all $\lambda \in[0,1]$. Let $P^{-}(0): X \rightarrow X_{1}$ and $P^{+}(0): X \rightarrow X_{2}$ denote the associated projections.

Let $\lambda \in[0,1]$ be arbitrary but fixed. For $\rho>0$, set

$$
U_{\rho, \lambda}:=U_{\rho}:=\left\{x \in X^{\alpha}:\left\|P^{-}(0)(x)\right\|_{\alpha}+\left\|P^{+}(0)(x)\right\|_{\alpha} \leq \rho\right\} .
$$

It follows from [5, Theorem 5.2.1] that $\operatorname{Inv}^{+}\left(U_{\rho}\right) \subset S$ provided that $\rho$ is small enough. Here, $S$ denotes the local stable manifold as defined in [5, Theorem 5.2.1]. It is tangent to $X_{2}$, which means that $\left\|x_{n}\right\|_{\alpha}^{-1} P\left(x_{n}\right)=\left\|x_{n}\right\|_{\alpha}^{-1}\left(x_{n}-\right.$ $\left.P^{+}(0)\left(x_{n}\right)\right) \rightarrow 0$ whenever $x_{n}$ is a sequence in $S \backslash\{0\}$ with $x_{n} \rightarrow 0$ in $X^{\alpha}$.

This proves that $\pi_{\lambda}$ is a sequence which satisfies Definition 2.2, so $\pi$ is indeed strongly linearizable.

Definition 2.4. Let $f(x):=x-a$ be defined in a neighbourhood of $a$ in $X^{\alpha}$. Then $\pi$ is called strongly linearizable in $a$ if $f[\pi]$ is strongly linearizable.

\section{Orientations and seeds}

Throughout this section, let $X$ be a metric space, $e \in X$, and $\pi$ a local semiflow defined in a neigborhood of $e$ in $X$ such that $\{e\}$ is an isolated invariant set admitting a strongly $\pi$-admissible isolating neighbourhood.

For $n \in \mathbb{N} \cup\{0\}, \mathcal{S}^{n}$ is an object of $[\mathcal{H} \mathcal{T}]$ (a connected simple system), which has itself only one object, namely $\left(D^{n} / S^{n-1},\left\{\left[S^{n-1}\right]\right\}\right)$, and exactly one morphism: the identity id: $\left(D^{n} / S^{n-1},\left\{\left[S^{n-1}\right]\right\}\right) \rightarrow\left(D^{n} / S^{n-1},\left\{\left[S^{n-1}\right]\right\}\right)$.

Definition 3.1. An $\left(n\right.$-)orientation is an ismorphism $o: \mathcal{S}^{n} \rightarrow \mathcal{C}(\pi, S)$ in $[\mathcal{H T}]$.

We will now develop a method which is based on continuous mappings $\mathbb{R}^{n} \rightarrow$ $X$ to obtain orientations or, depending on the point of view, to describe them. These mappings are called seeds, and they may or may not induce orientations.

Before defining them, we will introduce a few additional notational shortcuts: $A / B$ denotes the pair $(A / B,[B])$, that is, the explicit notation of the basepoint is omitted in order to keep certain diagrams readable. For every FM-index pair 
$\left(N_{1}, N_{2}\right)$ for $(\pi,\{e\})$, define $N_{2}^{-s}:=N_{2}^{-s}\left(N_{1}\right):=\left\{x \in N_{1}: \exists t \in[0, s], x \pi t \in\right.$ $\left.N_{2}\right\}$ and $N_{2}^{-\infty}:=N_{2}^{-\infty}\left(N_{1}\right):=\left\{x \in N_{1}: \exists t \in \mathbb{R}^{+}, x \pi t \in N_{2}\right\}$, that is, $N_{2}^{-\infty}=\bigcup_{s \in \mathbb{R}^{+}} N_{2}^{-s}$ (see also [2, Proposition 4.6]).

Definition 3.2. Let $n \in \mathbb{N} \cup\{0\}, U \subset \mathbb{R}^{n}, f: U \rightarrow X$ continuous with $f(0)=e$, and for every strongly $\pi$-admissible FM-index pair $\left(N_{1}, N_{2}\right)$ let there exist a $\lambda \in \mathbb{R}^{+}$such that $f^{\lambda}(x):=f(\lambda x)$ is defined for all $x \in D^{n}$ and $f^{\lambda}(x) \in$ $N_{2}^{-\infty}$ for all $x \in D^{n} \backslash\{0\}$. Then $f$ is called a seed for $(\pi, e)$.

It is not a priori clear whether seeds exist.

LEMma 3.3. Let $\left(N_{1}, N_{2}\right)$ be a strongly $\pi$-admissible FM-index pair for ( $\pi,\{e\}), \lambda \in \mathbb{R}^{+}$, and $f$ be a seed such that $f^{\lambda}(x)$ is defined for all $x \in D^{n}$ and $f^{\lambda}\left(D^{n}\right) \subset N_{1}$. Let $\Omega:=\left\{g: D^{n} \rightarrow N_{1}: g\right.$ is continuous and $\left.g(0)=e\right\}$ be equipped with the maximum metric. Then there is an $s \in \mathbb{R}^{+}$and a neighbourhood $U_{f^{\lambda}}$ of $f_{\mid D^{n}}^{\lambda}$ in $\Omega$ such that $g\left(D^{n}, S^{n-1}\right) \subset\left(N_{1}, N_{2}^{-s}\right)$ for all $g \in U_{f^{\lambda}}$.

Proof. Let $\tau(x, g):=\sup \left\{t \in \mathbb{R}^{+}: g(x) \pi t \in \operatorname{cl}\left(N_{1} \backslash N_{2}\right)\right\}$. We have $\tau\left(x, f^{\lambda}\right)<\infty$ for all $x \in S^{n-1}$ because $f$ is a seed.

Let $x \in S^{n-1}$ and $\left.\left.\varepsilon \in\right] 0,1\right]$ with $f^{\lambda}(x) \pi(\tau(x)+\varepsilon) \in X \backslash \operatorname{cl}\left(N_{1} \backslash N_{2}\right)$. Since $X \backslash \operatorname{cl}\left(N_{1} \backslash N_{2}\right)$ is an open set, there exist neighbourhoods $V_{x}$ of $x$ in $D^{n}$ and $U_{x, f^{\lambda}}$ of $f_{\mid D^{n}}^{\lambda} \in \Omega$ such that $g(x) \pi(\tau(x)+\varepsilon) \notin \operatorname{cl}\left(N_{1} \backslash N_{2}\right)$ for all $(x, g) \in V_{x} \times U_{x, f^{\lambda}}$, showing that $\tau(\xi, g) \leq \tau(x)+\varepsilon \leq \tau(x)+1$ for all $(\xi, g) \in V_{x} \times U_{x, f^{\lambda}}$.

Due to the compactness of $S^{n-1}$, there are $x_{1}, \ldots, x_{n} \in S^{n-1}$ such that $S^{n-1} \subset \bigcup_{k=1, \ldots, n} V_{x_{k}}$. Letting $\widetilde{U}_{f^{\lambda}}:=\bigcap_{k=1, \ldots, n} U_{x_{k}, f^{\lambda}}$, it follows that $\tau(x, g) \leq$ $\max _{k=1, \ldots, n} \tau\left(x_{k}, f^{\lambda}\right)+1=: s$ for all $(x, g) \in S^{n-1} \times \widetilde{U}_{f^{\lambda}}$. Hence, for every $(x, g) \in$ $S^{n-1} \times \widetilde{U}_{f^{\lambda}}$ we have $g(x) \in N_{1}$ and $g(x) \pi r \notin N_{1}$ for some $r \in[0, s]$, showing that $g(x) \in N_{2}^{-s}$.

Lemma 3.4. Let $f: U \rightarrow X, U \subset \mathbb{R}^{n}$, be continuous with $f(0)=e$, and suppose that there exist a strongly $\pi$-admissible FM-index pair $\left(N_{1}, N_{2}\right)$ for $(\pi,\{e\})$ and $a \lambda \in \mathbb{R}^{+}$such that $f^{\lambda}(x)$ is defined for all $x \in D^{n}$ and $f^{\lambda}\left(D^{n} \backslash\{0\}\right) \subset N_{2}^{-\infty}$. Then $f$ is a seed.

Proof. Let $\left(M_{1}, M_{2}\right)$ be a strongly $\pi$-admissible FM-index pair for $(\pi,\{e\})$ and let $x \in D^{n} \backslash\{0\}$. By our assumptions, there exists a strongly $\pi$-admissible FM-index pair $\left(N_{1}, N_{2}\right)$ for $(\pi,\{e\})$ and a $\lambda \in \mathbb{R}^{+}$with $f^{\lambda}\left(D^{n} \backslash\{0\}\right) \subset N_{2}^{-\infty}$. The set $N:=\operatorname{cl}\left(N_{1} \backslash N_{2}\right)$ is an isolating neighbourhood for $(\pi,\{e\})$, and $\left(\widetilde{N}_{1}, \widetilde{N}_{2}\right)$ $:=\left(N_{1} \cap N, N_{2} \cap N\right)$ is again a strongly admissible FM-index pair.

By the continuity of $f$ and because $e \in \operatorname{int} N$, there is a $\widetilde{\lambda} \in] 0, \lambda]$ such that $f^{\tilde{\lambda}}\left(D^{n}\right) \subset \widetilde{N}_{1}$. We have $N_{1} \backslash N_{2}=\widetilde{N}_{1} \backslash \widetilde{N}_{2}$, showing that $f^{\tilde{\lambda}}\left(D^{n} \backslash\{0\}\right) \subset \widetilde{N}_{2}^{-\infty}$.

It follows from [2, Lemma 4.8] that there are an $s \in \mathbb{R}^{+}$and a strongly $\pi$-admissible FM-index pair $\left(L_{1}, L_{2}\right)$ for $(\pi,\{0\})$ such that $L_{1}$ is an isolating 
neighbourhood for $(\pi,\{e\})$ and

$$
\left(M_{1}, M_{2}\right) \subset\left(M_{1}, M_{2}^{-s}\right) \supset\left(L_{1}, L_{2}\right) \subset\left(\widetilde{N}_{1}, \widetilde{N}_{2}^{-s}\right) \supset\left(\widetilde{N}_{1}, \widetilde{N}_{2}\right) .
$$

We can choose $\tilde{\widetilde{\lambda}} \in] 0, \tilde{\lambda}]$ such that $f^{\tilde{\tilde{\lambda}}}\left(D^{n}\right) \subset L_{1}$.

For every $x \in D^{n} \backslash\{0\}$, it follows that $f^{\tilde{\tilde{\lambda}}}(x) \pi t \notin \widetilde{N}_{1} \supset L_{1}$ for some $t \in \mathbb{R}^{+}$ because $\widetilde{N}_{1}$ is an isolating neighbourhood and $f^{\tilde{\lambda}}(x) \in \widetilde{N}_{2}^{-\infty}$ for all $x \in D^{n} \backslash\{0\}$. Hence, there exists an $r \in[0, t]$ with $f^{\tilde{\tilde{\lambda}}}(x) \pi r \in L_{2}$, showing that $f^{\tilde{\tilde{\lambda}}}(x) \in$ $M_{2}^{-\infty}$.

Definition 3.5. Let $\left(N_{1}, N_{2}\right)$ be a strongly $\pi$-admissible FM-index pair for $(\pi,\{e\}), f$ a seed, and $\lambda \in \mathbb{R}^{+}$such that $f^{\lambda}(x)$ is defined for all $x \in D^{n}$ and $f^{\lambda}\left(D^{n} \backslash\{0\}\right) \subset N_{2}^{-\infty} \cdot \bar{f}:=\bar{f}_{N_{1}, N_{2}}: D^{n} / S^{n-1} \rightarrow N_{1} / N_{2}$ denotes the unique morphism in $\mathcal{H} \mathcal{T}$ for which

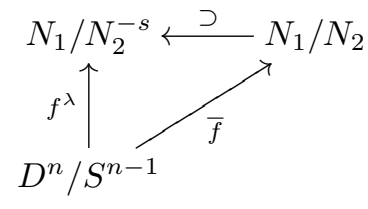

commutes whenever $f^{\lambda}\left(S^{n-1}\right) \subset N_{2}^{-s}, s \in \mathbb{R}^{+}$.

The subscript of $\bar{f}$, although important, is often omitted when the FM-index pair is clear from the context.

Definition 3.6. Let $f$ be a seed for $(\pi, e)$, and let $\langle f, \pi, e\rangle: \mathcal{S}^{n} \rightarrow \mathcal{C}(\pi,\{e\})$ denote the morphism in $[\mathcal{H} \mathcal{T}]$ for which

$$
\langle f, \pi, e\rangle\left(\left(D^{n} / S^{n-1},\left\{\left[S^{n-1}\right]\right\}\right),\left(N_{1} / N_{2},\left\{\left[N_{2}\right]\right\}\right)\right)=\bar{f}_{N_{1}, N_{2}}
$$

whenever $\left(N_{1}, N_{2}\right)$ is a strongly admissible FM-index pair for $(\pi,\{e\})$. Since $e=f(0)$ by the assumption of $f$ being a seed, we will also write $\langle f, \pi\rangle$.

Lemma 3.7. Let $\left(N_{1}, N_{2}\right)$ be a strongly admissible FM-index pair for $(\pi,\{e\})$. Then $\bar{f}: D^{n} / S^{n-1} \rightarrow N_{1} / N_{2}$ is well-defined.

Proof. There are two parameters involved in Definition 3.5, $s$ and $\lambda$. Firstly, we will consider $s$. Given $r, s \in \mathbb{R}^{+}$with $f^{\lambda}\left(S^{n-1}\right) \subset N_{2}^{-r} \subset N_{2}^{-s}$, there is a commutative diagram

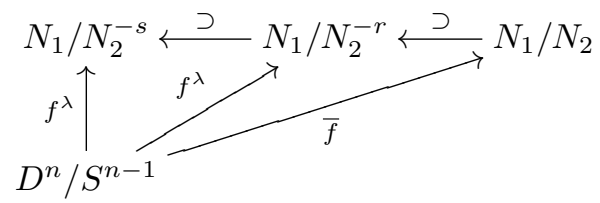

showing that $r$ and $s$ induce the same morphism $\bar{f}$. 
Secondly, it follows from Lemma 3.3 that, for every $\mu \in] 0, \lambda]$ there are an $s \in \mathbb{R}^{+}$and a neighbourhood $U$ of $f_{\mid D^{n}}^{\mu}$ in $\Omega$ such that

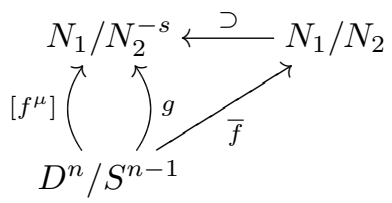

is defined for all $g \in U$ and commutative whenever $g$ is homotopic to $\left[f^{\mu}\right]$. Since $f^{\widetilde{\mu}}\left(D^{n}\right) \subset f^{\mu}\left(D^{n}\right) \subset N_{1}$ for all $\widetilde{\mu} \leq \mu$, one has $f^{\widetilde{\mu}} \in U$ for all $\widetilde{\mu} \leq \mu$ large enough. Hence, $\mu \mapsto \bar{f}^{\mu} N_{1}, N_{2}$ is locally constant on $\left.] 0, \lambda\right]$, which is connected.

Using [9, Proposition I.8.2], it is easy to give a direct formula for $\bar{f}$. Let $f$ be a seed for $(\pi, e),\left(N_{1}, N_{2}\right)$ be a strongly admissible FM-index pair for $(\pi,\{e\})$, and $\lambda \in \mathbb{R}^{+}$be sufficiently small that $f^{\lambda}\left(D^{n}\right)$ is defined and $f^{\lambda}\left(D^{n}\right) \subset N_{1}$. Then, $\bar{f}=[g]_{\mathcal{H} \mathcal{T}}$ where $g: D^{n} / S^{n-1} \rightarrow N_{1} / N_{2}$,

$$
g([x]):= \begin{cases}{\left[f^{\lambda}(x) \pi s\right]} & f^{\lambda}(x) \pi[0, s] \text { is defined and } f^{\lambda}(x) \pi[0, s] \subset N_{1} \backslash N_{2}, \\ {\left[N_{2}\right]} & \text { otherwise. }\end{cases}
$$

Lemma 3.8. Let $\Omega:=\left\{g: D^{n} \rightarrow X: g\right.$ is a seed for $\left.(\pi, e)\right\}$ be equipped with the maximum metric. Then $g \mapsto \bar{g}$, is constant on path components of $\Omega$.

Proof. Let $\lambda \mapsto g_{\lambda},[0,1] \rightarrow \Omega$ be continuous. It is sufficient to show that $g \mapsto \bar{g}$ is locally constant.

Let $\left(N_{1}, N_{2}\right)$ be a strongly admissible FM-index pair for $(\pi,\{e\})$ and $\lambda_{0} \in$ $[0,1]$. There exists a $\mu>0$ such that

$$
g_{\lambda_{0}}^{\mu}\left(D^{n}\right) \subset \operatorname{int}\left(N_{1} \backslash N_{2}\right) .
$$

Hence, there is a neighbourhood $U$ of $g_{\lambda_{0}}$ in $\Omega$ such that

$$
h^{\mu}\left(D^{n}\right) \subset \operatorname{int}\left(N_{1} \backslash N_{2}\right)
$$

for all $h \in U$. By Lemma 3.3, there is another neighbourhood $\widetilde{U} \subset U$ of $g_{\lambda_{0}}$ in $\Omega$ and an $s \in \mathbb{R}^{+}$such that

$$
h^{\mu}\left(S^{n-1}\right) \subset N_{2}^{-s}
$$

for all $h \in \widetilde{U}$. The continuity of $\lambda \mapsto g_{\lambda}$ now implies that there is a neighbourhood of $V$ of $\lambda_{0}$ in $[0,1]$ such that $g_{\lambda} \in \widetilde{U}$ for all $\lambda \in V$, so for $\lambda \in V$

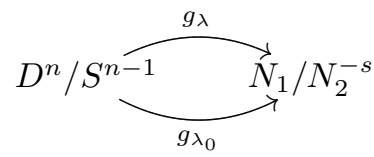

is defined and commutative. This implies that $\overline{g_{\lambda}}$ is constant on $V$. 
LEMmA 3.9. Let $\left(N_{1}, N_{2}\right)$ and $\left(M_{1}, M_{2}\right)$ be strongly admissible FM-index pairs for $(\pi,\{e\})$ and $f$ a seed. Then

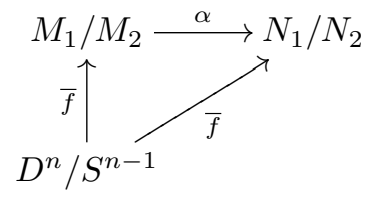

commutes, where $\alpha$ denotes the inner morphism of the categorial Conley index.

Proof. In view of [2, Lemma 4.8], it is sufficient to prove our claim in the special case $\left(M_{1}, M_{2}\right) \subset\left(N_{1}, N_{2}\right)$. It follows immediately from the definitions of $M_{2}^{-s}$ and $N_{2}^{-s}$ that $M_{2}^{-s} \subset N_{2}^{-s}$ for all $s \in \mathbb{R}^{+}$.

By Lemma 3.3, we may choose $s \in \mathbb{R}^{+}$and $\lambda \in[0,1]$ such that

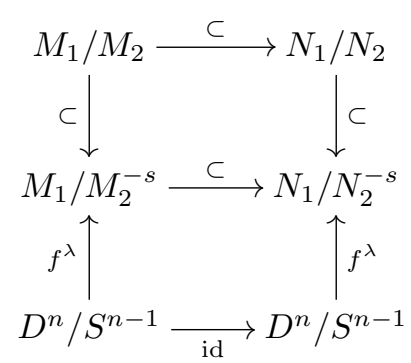

is defined and commutative. Consequently, composing the vertical arrows,

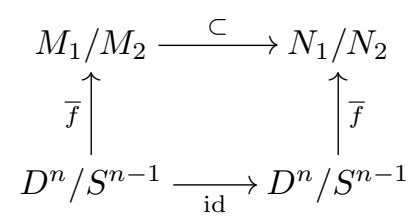

commutes by Definition 3.5.

Proposition 3.10. Let $\left(\pi_{k}\right)_{k \in \mathbb{N} \cup\{\infty\}}$ be a family of semiflows such that $\pi_{k} \rightarrow \pi_{\infty}:=\pi$ and let $\left(N_{1, \infty}, N_{2, \infty}\right),\left(\widetilde{N}_{1, \infty}, \widetilde{N}_{2, \infty}\right)$ be strongly $\pi_{\infty}$-admissible FM-index pairs for $\left(\pi_{\infty},\{e\}\right)$ such that $N_{1, \infty}$ is a strongly admissible isolating neighbourhood for $\left(\pi_{\infty},\{e\}\right)$. Further, let $\left(N_{1, k}, N_{2, k}\right)_{k \in \mathbb{N}},\left(\tilde{N}_{1, k}, \tilde{N}_{2, k}\right)_{k \in \mathbb{N}}$ be families of strongly $\pi_{k}$-admissible FM-index pairs for $\left(\pi_{k},\{e\}\right)$ such that

$$
\left(\tilde{N}_{1, k}, \tilde{N}_{2, k}\right) \subset\left(\tilde{N}_{1, \infty}, \tilde{N}_{2, \infty}\right) \subset\left(N_{1, k}, N_{2, k}\right) \subset\left(N_{1, \infty}, N_{2, \infty}\right)
$$

for all $k \in \mathbb{N}$. Finally, let $f: D^{n} \rightarrow X$ be a common seed, that is, for every $k \in \mathbb{N} \cup\{\infty\}$ it holds that $f$ is a seed for $\left(\pi_{k}, e\right)$. Then there is an $n_{0} \in \mathbb{N}$ such 
that

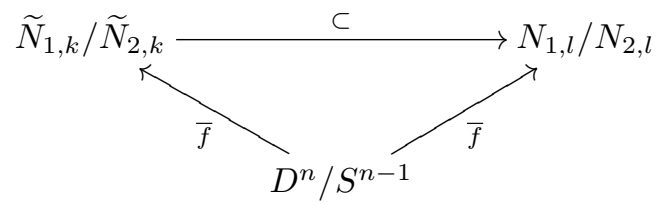

is commutative for all $k, l \in \mathbb{N} \cup\{\infty\}$ with $k, l \geq n_{0}$.

Lemma 3.11. In addition to the hypothesis of Proposition 3.10 let $\lambda \in] 0,1]$ such that $f^{\lambda}\left(D^{n}\right) \subset N_{1, k}$ and $r \in \mathbb{R}^{+}$. Then:

(a) $M:=M_{\lambda, r}:=\left\{(x, s) \in S^{n-1} \times[0, r]: f^{\lambda}(x) \pi[0, s] \subset \widetilde{N}_{1, \infty}\right\}$ is compact.

(b) $g:=g_{\lambda}:[0, r] \times D^{n} \rightarrow \widetilde{N}_{1, \infty} / \widetilde{N}_{2, \infty}$,

$g(s, x):= \begin{cases}f^{\lambda}(x) \pi s & f^{\lambda}(x) \pi_{\infty}[0, s] \text { is defined and } f^{\lambda}(x) \pi[0, s] \subset \widetilde{N}_{1, \infty}, \\ {\left[\widetilde{N}_{2, \infty}\right]} & \text { otherwise, }\end{cases}$

is continuous.

(c) There is a $\tau \in \mathbb{R}^{+}$such that $g\left([0, r] \times S^{n-1}\right) \subset N_{2, k}^{-\tau} / \widetilde{N}_{2, \infty}$ for all $k \in \mathbb{N} \cup\{\infty\}$ sufficiently large.

Proof. (a) $S^{n-1} \times[0, r]$ is compact, so it suffices to prove that $M$ is closed. Let $\left(x_{k}, s_{k}\right) \rightarrow\left(x_{0}, s_{0}\right)$ in $M$ and $s \in\left[0, s_{0}\right.$ [. It follows that for all $k \in \mathbb{N}$ large enough $s_{k}>s$, so $x_{k} \pi s \in \widetilde{N}_{1, \infty}$ and $x_{0} \pi s \in \widetilde{N}_{1, \infty}$. Hence, by the closedness of $\widetilde{N}_{1, \infty}$, we have $x_{0} \pi\left[0, s_{0}\right] \subset \widetilde{N}_{1, \infty}$, and thus $\left(x_{0}, s_{0}\right) \in M$.

(b) This follows from [9, Proposition I.8.1].

(c) Let $\widetilde{M}:=\left\{\left(f^{\lambda}(x), s\right):(x, s) \in M\right\}, x \in \pi(\widetilde{M})$, and note that $\pi(\widetilde{M}) \subset$ $\widetilde{N}_{1, \infty} \subset N_{1, k}$ for all $k \in \mathbb{N}$. By the assumption that $N_{1, \infty}$ is a (strongly admissible) isolating neighbourhood of $\{e\}$ relative to $\pi$, there is a $t=t_{x} \in \mathbb{R}^{+}$such that $f^{\lambda}(x) \pi t \in X \backslash N_{1, \infty}$. Otherwise, there would be a full solution of $\pi$ lying entirely in $N_{2, \infty}$ (using the strong admissibility), contradicting the assumption of $N_{1, \infty}$ being an isolating neighbourhood.

Hence, there are $n_{0}=n_{0}(x)$ and a neighbourhood $U_{x}$ of $x$ in $\pi(\widetilde{M})$ such that $U_{x} \pi_{k} t \in X \backslash N_{1, \infty} \subset X \backslash N_{1, k}$ for all $k \geq n_{0}$. Consequently, for every $x \in U_{x}$, there is an $r \in\left[0, t_{x}\right]$ with $x \pi_{k} r \in N_{2, k}$. The compactness of $\pi(\widetilde{M})$ implies that there are $x_{1}, \ldots, x_{N} \in \pi(\widetilde{M})$ with $\pi(\widetilde{M}) \subset \bigcup_{i=1, \ldots, N} U_{x_{i}}$. We can choose $\tau:=\max _{i=1, \ldots, N} t_{x_{i}}$ and $n_{0}:=\max _{i=1, \ldots, N} n_{0}\left(x_{i}\right)$.

For every $(s, x) \in D(g)$ one has either $g(s, x) \in \pi(\widetilde{M})$ or $g(s, x)=\left[\widetilde{N}_{2, \infty}\right]$, so $g(s, x) \in N_{2, k}^{-\tau} / \widetilde{N}_{2, \infty}$ for all $k \geq n_{0}$.

Proof of Proposition 3.10. Let $\tau \in \mathbb{R}^{+}$be given by Lemma 3.11, and assume that $f^{\lambda}\left(S^{n-1}\right) \subset N_{2, \infty}^{-s}$ for $\lambda \in[0,1]$ and $s \in \mathbb{R}^{+}$. It follows that there is 
an $n_{0} \in \mathbb{N}$ such that for all $k \geq n_{0}$

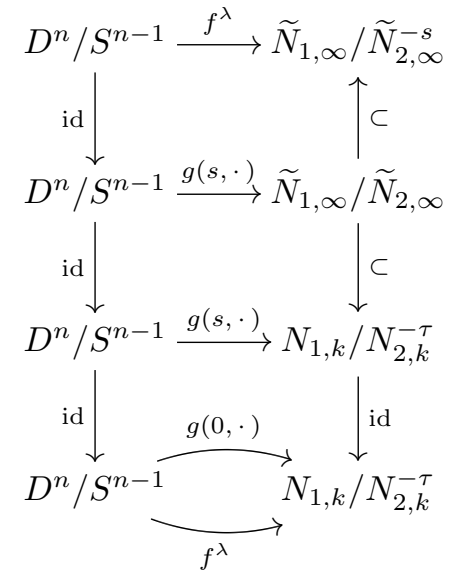

commutes in $\mathcal{H} \mathcal{T}$. This shows that

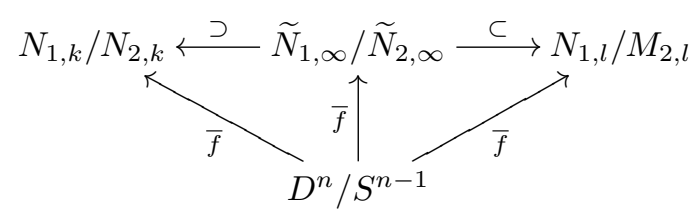

commutes for all $k, l \geq n_{0}$. It follows from Lemma 3.9 that

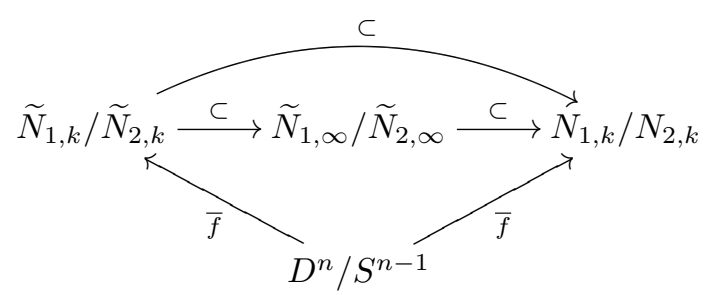

is commutative and thus also

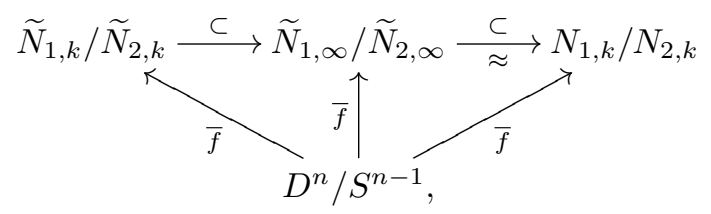

where $\approx$ indicates an isomorphism. Finally, we conclude that

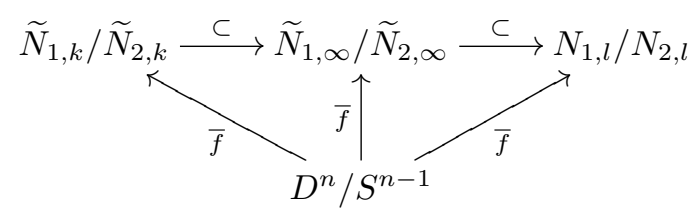

commutes for all $k, l \geq n_{0}$. 
THEOREM 3.12. Let $\Lambda$ be a connected metric space and let $\left(\pi_{\lambda},\{e\}\right)_{\lambda \in \Lambda}$ be an $\mathcal{S}$-continuous family such that there exists a common seed $f: D^{n} \rightarrow X$. If there exists a $\lambda_{0} \in \Lambda$ such that $\left\langle f, \pi_{\lambda_{0}}, e\right\rangle$ is an isomorphism, then $\left\langle f, \pi_{\lambda}, e\right\rangle$ is an isomorphism for all $\lambda \in \Lambda$.

Proof. Let $\chi: \Lambda \rightarrow\{0,1\}$ be defined by

$$
\chi(\lambda):= \begin{cases}1 & \left\langle f, \pi_{\lambda}, e\right\rangle \text { is an isomorphism, } \\ 0 & \text { otherwise. }\end{cases}
$$

It follows from [9, Theorem I.12.3] and Proposition 3.10 that $\chi$ is locally constant on $\Lambda$, which is a connected metric space.

\section{Orientation for fixed points of linearizable semiflows}

Throughout this section, let $X$ be a Banach space and $\pi, \pi^{\prime}$ be strongly linearizable semiflows defined in a neighbourhood of 0 . Moreover, suppose that $\pi^{\prime}$ is a linear semiflow, and let $n=\operatorname{dim} X_{1}$. Recall that the subspaces $X_{1}=$ $P_{\pi} X$ depends on the semiflow $\pi$. We will use the notation introduced in the Preliminaries section.

Lemma 4.1. Let $f: D^{n} \rightarrow X$ be continuous, $f(0)=0$, and $0<\theta \in \mathbb{R}^{+}$such that

$$
\left\|P_{\pi} \circ f(x)\right\|>\theta\|f(x)\|
$$

for all $x \neq 0$ in a sufficiently small neighbourhood of 0 . Then $f$ is a seed for $(\pi, 0)$.

Proof. Suppose that $f$ is not a seed. By Definition 2.2, there exists a neighbourhood $U$ of 0 such that

$$
\left\|y_{n}\right\|^{-1} P_{\pi}\left(y_{n}\right) \rightarrow 0
$$

whenever $y_{n}$ is a sequence in $\operatorname{Inv}^{+}(U) \backslash\{0\}$ with $x_{n} \rightarrow 0$ as $n \rightarrow \infty$.

Let $\left(N_{1}, N_{2}\right)$ be a strongly admissible FM-index pair with $N_{1} \subset U$. By Lemma 3.4, there is a sequence $0 \neq x_{n} \rightarrow 0$ such that $f\left(x_{n}\right) \in \operatorname{Inv}^{+}\left(N_{1}\right) \subset$ $\operatorname{Inv}^{+}(U)$ for all $n \in \mathbb{N}$.

We have $f\left(x_{n}\right) \neq 0$ for all $n \in \mathbb{N}$ by (4.1). Since $\pi$ is strongly linearizable, it follows from (4.2) that

$$
\left\|f\left(x_{n}\right)\right\|^{-1} P_{\pi} \circ f\left(x_{n}\right) \rightarrow 0,
$$

a contradiction to (4.1).

Remark 4.2. (4.1) holds if $f(x) \in X_{1}$ for all $x \in X$. Moreover, (4.1) also holds if $f$ has a Fréchet-derivative $D f(0)$ at 0 with $\operatorname{ker}(P \circ D f(0))=\{0\}$. 
COROLlary 4.3. Let $f: D^{n} \rightarrow X_{1}$ be continuous and injective with $f(0)=0$. Further, let $\Lambda$ be a connected metric space and let $\left(\pi_{\lambda}\right)_{\lambda \in \Lambda}$ be an $\mathcal{S}$-continuous family of strongly linearizable semiflows with $X_{1}=X_{1}\left(\pi_{\lambda}\right)$ being constant. Then $f$ is a seed for $\left(\pi_{\lambda}, 0\right)$ for all $\lambda \in \Lambda$. Furthermore, if there is a $\lambda_{0} \in \Lambda$ such that $\left\langle f, \pi_{\lambda_{0}}, 0\right\rangle$ is an isomorphism, then $\left\langle f, \pi_{\lambda}, 0\right\rangle$ is an isomorphism for all $\lambda \in \Lambda$.

Proof. $f$ is a seed for every $\pi_{\lambda}$ by Lemma 4.1 and the remark thereafter. Thus, the claim follows from Theorem 3.12.

Proposition 4.4. Let $\left(N_{1}, N_{2}\right)$ be a strongly $\pi^{\prime}$-admissible FM-index pair for $\left(\pi^{\prime},\{0\}\right)$, and let $f: D^{n} \rightarrow X_{1}$ be injective and continuous with $f(0)=0$. Then $\bar{f}: D^{n} / S^{n-1} \rightarrow N_{1} / N_{2}$ is an isomorphism in the homotopy category of pointed spaces.

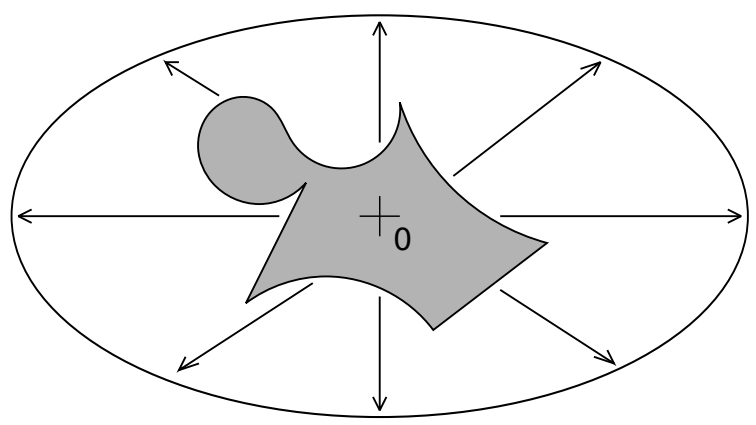

Figure 1. Homotopy of a seed

Proof. It is shown in the proof of [9, Theorem I.11.1] that there exists an isolating block $B=B_{1} \oplus B_{2}$ with

$$
B_{1}=\left\{x \in X_{1}: V^{+}(x) \leq 1\right\}, \quad B_{2}=\left\{x \in X_{2}: V^{-}(x) \leq 1\right\}
$$

and $B^{-}=\partial B_{1} \oplus B_{2} . B_{1} / \partial B_{1}$ is a strong deformation retraction of $B / B^{-}$, that is, the inclusion induced mapping

$$
\left(B_{1} / \partial B_{1},\left[B_{1}\right]\right) \stackrel{\subset}{\longrightarrow}\left(B / B^{-},\left[B^{-}\right]\right)
$$

is an isomorphism in the homotopy category of pointed spaces.

There exists a $\lambda \in] 0,1]$ such that $f^{\lambda}$ is injective and $f^{\lambda}\left(D^{n}\right) \subset \operatorname{int} B_{1}$. Moreover, there is a continuous functional $\rho: D^{n} \backslash\{0\} \rightarrow \mathbb{R}^{+}$with $f^{\lambda}(x) \pi^{\prime}(\rho(x)) \in$ $\partial B_{1}$ for all $x \in D^{n} \backslash\{0\}$ (see [9, Lemma 3.8]).

Define $g:[0,1] \times D^{n} \rightarrow X_{1}$ by

$$
g(\mu, x):= \begin{cases}f^{\lambda}(x) \pi^{\prime}(\mu \kappa(x) \rho(x)), & x \neq 0, \\ 0, & x=0,\end{cases}
$$


where $\kappa: D^{n} \rightarrow[0,1]$ is continuous, $\kappa(x)=1$ for all $x \in S^{n-1}$, and there is a neighbourhood $U$ of 0 in $D^{n}$ with $\kappa(x)=0$ for all $x \in U$. This is illustrated in Figure 1: the grey area shows the image of $f^{\lambda}$, the arrows indicate the flow on $B_{1}$. Lemma 4.1 and the remark thereafter imply that $g(\mu, \cdot)$ is a seed for every $\mu \in[0,1]$. It follows from Lemma 3.8 that $\bar{f}=\overline{g(0, \cdot)}=\overline{g(1, \cdot)}$.

Both spaces, $D^{n} / S^{n-1}$ and $B_{1} / \partial B_{1}$ are homeomorphic to $S^{n}$. Let $h$ be induced by the following commutative diagram in the category of pointed spaces, where the vertical arrows denote isomorphisms:

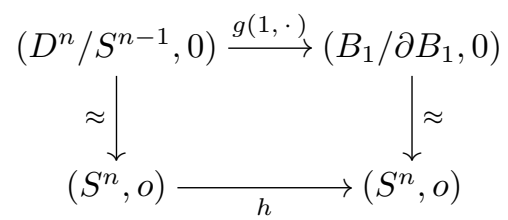

$o \in S^{n}$ can be chosen arbitrary as long as the morphisms are basepoint-preserving.

We now have $h^{-1}(\{o\})=\{o\}$. Since $\kappa(x)=0$ in a neighbourhood of 0 , and by the injectivity of $f$, there is an open neighbourhood of $V$ of $o$ in $S^{n}$ such that $h_{\mid V}$ is injective. $h(V)$ is open by the invariance of domain, so $h$ is a local homeomorphism at $o$. Therefore, $\operatorname{deg} h= \pm 1$ by [4, Proposition 2.2.30]. It follows that $[h]_{\mathcal{H} \mathcal{T}}$ is an isomorphism (see [12, Theorem VIII.10.1]). Therefore, $\bar{f}=[g(1, \cdot)]_{\mathcal{H} \mathcal{T}}$ is also an isomorphism.

It is now straightforward to formulate the following

Proposition 4.5. Let $f: D^{n} \rightarrow X_{1}, f(0)=0$, be injective and continuous. Then $f$ is a seed for $(\pi, 0)$, and $\langle f, \pi, 0\rangle$ is an orientation for $(\pi, 0)$.

Proof. Since $\pi$ is strongly linearizable, there is an $\mathcal{S}$-continuous family $\left(\pi_{\lambda},\{0\}\right)$ with $\pi_{1}=\pi$ and $\pi^{\prime}:=\pi_{0}$ being linear.

It follows from Proposition 4.4 that $\left\langle f, \pi^{\prime}, 0\right\rangle$ is an isomorphism. Using Corollary 4.3 and the definition of strong linearizability, one obtains that $\langle f, \pi, 0\rangle$ is also an isomorphism.

Corollary 4.6. Let $f: D^{n} \rightarrow X$ with $f(0)=0$. Assume that the Fréchetderivative $D f(0)$ exists and $P \circ D f(0): \mathbb{R}^{n} \rightarrow X_{1}$ is an isomorphism. Then $f$ and $P \circ D f(0)$ are seeds for $(\pi, 0)$, and $\langle f, \pi, 0\rangle=\langle P \circ D f(0), \pi, 0\rangle$ are orientations.

Proof. By Lemma 4.1, $g_{\lambda}: D^{n} \rightarrow X$,

$$
g_{\lambda}(x):=\lambda f(x)+(1-\lambda)(P \circ D f(0)) x,
$$

is a seed for every $\lambda \in[0,1]$. We have $g_{0}=f, g_{1}=P \circ D f(0)$, so it follows from Lemma 3.8 that $\overline{g_{\lambda}}$ is constant.

Finally, $\langle P \circ D f(0), \pi, 0\rangle$ is an orientation by Proposition 4.5. 
One might expect that an orientation is only a choice of a basis for $X_{1}$. The relationship between orientations (induced by the above seeds) and bases is established by the following proposition, which states that compatible bases induce the same orientation and vice versa.

Proposition 4.7. Let $\Phi_{1}, \Phi_{2} \in \mathcal{L}\left(\mathbb{R}^{n}, X_{1}\right)$ be ismorphisms. Then $\left\langle\Phi_{1}, \pi, e\right\rangle$ $=\left\langle\Phi_{2}, \pi, e\right\rangle$ if and only if $\operatorname{det} \Phi_{2}^{-1} \Phi_{1}>0$.

Let $E$ and $F$ be finite-dimensional normed spaces. For $A, B \in \operatorname{ISO}(E, F)$ let $A \sim B$ (homotopic) if and only if there exists a family $\left(C_{\lambda}\right)_{\lambda \in[0,1]}$ in $\operatorname{ISO}(E, F)$ such that

(1) $C_{0}=A$;

(2) $C_{1}=B$;

(3) $\lambda \mapsto C_{\lambda}$ is continuous

It is well known [8, Proposition 9.36] that $A \sim B$ if and only if $\operatorname{det} A \cdot \operatorname{det} B>0$.

Proof. The case $n=0$ is trivial, so we may assume that $n \geq 1$. Suppose that $\operatorname{det} \Phi_{2}^{-1} \Phi_{1}>0$. Then, there exists $H \in C\left([0,1], \operatorname{ISO}\left(\mathbb{R}^{n}, X_{1}\right)\right)$ such that $H(0, \cdot)=\Phi_{1}$ and $H(1, \cdot)=\Phi_{2}$.

It follows from Lemma 4.1 that $H(\lambda, \cdot)$ is a seed for all $\lambda \in[0,1]$ and from Lemma 3.8 that $\left\langle\Phi_{1}, \pi, 0\right\rangle=\left\langle\Phi_{2}, \pi, 0\right\rangle$.

In order to prove the only-if part, it is sufficient to show that there are $\Phi_{1}$, $\Phi_{2}$ with $\left\langle\Phi_{1}, \pi, 0\right\rangle \neq\left\langle\Phi_{2}, \pi, 0\right\rangle$. Let $\Phi_{1} \in \operatorname{ISO}\left(\mathbb{R}^{n}, X_{1}\right)$ be arbitrary and define $\Phi_{2}\left(x_{1}, \ldots, x_{n}\right):=\Phi_{1}\left(-x_{1}, x_{2}, \ldots, x_{n}\right)$ so that $\operatorname{det} \Phi_{2}^{-1} \Phi_{1}=-1$. Further, let $\left(N_{1}, N_{2}\right)$ be a strongly $\pi$-admissible FM-index pair for $\left.\left.(\pi,\{0\}), \lambda \in\right] 0,1\right]$, and $s \in \mathbb{R}^{+}$such that $\Phi_{1}^{\lambda}\left(D^{n}, S^{n-1}\right) \subset\left(N_{1}, N_{2}^{-s}\right)$,

Setting $\alpha\left(x_{1}, \ldots, x_{n}\right):=\left(-x_{1}, x_{2}, \ldots, x_{n}\right)$, it follows that

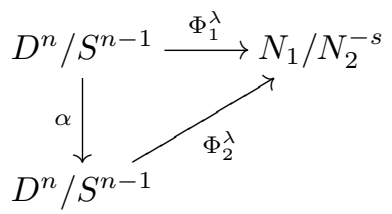

is a commutative diagram in the category of pointed topological spaces. Hence, passing (4.3) to singular homology, we obtain $-1=H_{n}(\alpha)=H_{n}\left(\overline{\Phi_{2}^{\lambda}}\right)^{-1} \circ H_{n}\left(\overline{\Phi_{1}^{\lambda}}\right)$ (see [4, Section 2.2] for the computation of $\left.H_{q}(\alpha)\right)$. This shows that $\left\langle\Phi_{1}, \pi, 0\right\rangle \neq$ $\left\langle\Phi_{2}, \pi, 0\right\rangle$.

\section{The effect of homeomorphisms}

Definition 5.1. For every $q \in \mathbb{Z}$, let $\mu_{q}: \mathbb{Z} \rightarrow H_{q}\left(\mathcal{S}^{q}\right)$ be an isomorphism and $\mu:=\left(\mu_{q}\right)_{q \in \mathbb{Z}}$. Then, given an arbitrary morphism $f: H_{q+k}\left[\mathcal{S}^{q+k}\right] \rightarrow H_{q}\left(\mathcal{S}^{q}\right)$, $k \in \mathbb{Z}$, there is a unique number $\theta(f):=\theta(f, \mu, q, k)$ such that $f \circ \mu_{q+k}=$ $\theta(f, \mu, q, k) \cdot \mu_{q}$. 
Until further notice, we will work with a fixed but arbitrary collection $\mu$ of isomorphisms.

Let $X$ and $Y$ be Banach spaces and let $\pi$ be a strongly linearizable local semiflow on $X$. As in the previous section, let $X_{1}=X_{1}(\pi)$ be defined as in the definition of strong linearizability and choose $n:=\operatorname{dim} X_{1}$. Let $U \subset X$ be a neighbourhood of 0 in $X, V \subset Y$ and $f: U \rightarrow V$ a homeomorphism. Using orientations $o_{1}: \mathcal{S}^{n} \rightarrow \mathcal{C}(\pi,\{0\})$ and $o_{2}: \mathcal{S}^{n} \rightarrow \mathcal{C}(f[\pi],\{f(0)\})$, the action of $f$ can be described by its induced action $f^{*}$ on $\mathcal{S}^{n}$, whose singular homology can be expressed by a number $\theta \in \mathbb{Z}$.

Definition 5.2. Let $o_{1}, o_{2}$ be orientations for $(\pi, 0)$, resp. $(f[\pi], f(0))$. $f^{*}:=f_{o_{1}, o_{2}}^{*}$ (we drop the subscript when no confusion can arise) denotes the unique morphism in $\mathcal{H} \mathcal{T}$ for which

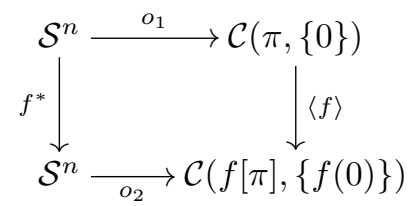

is commutative. Moreover, let $\theta(\langle f\rangle):=\theta\left(\langle f\rangle, \mu, o_{1}, o_{2}\right):=\theta\left(H_{n}\left(f_{o_{1}, o_{2}}^{*}\right), \mu, n, 0\right)$.

In general, the morphism $H_{q}\left(f^{*}\right)$ depends on $o_{1}$ and $o_{2}$. However, if we assume that $X=Y, f(0)=0, f$ is Fréchet-differentiable in 0 , and $D f(0)$ is an isomorphism, then $H_{q}\left(f^{*}\right)$ depends only on $D f(0)$ :

Proposition 5.3. Suppose that $D f(0)=\mathrm{id}_{X}$, and let $o: D^{n} \rightarrow X_{1}$ be injective and continuous with $o(0)=0$. Then:

(a) $o$ is a seed for $\pi$ and $f[\pi]$;

(b) $\langle o, \pi\rangle$ and $\langle o, f[\pi]\rangle$ are orientations;

(c) $\theta(\langle f\rangle, \mu,\langle o, \pi\rangle,\langle o, f[\pi]\rangle)=1$.

Proof. Letting $g_{r}(x):=r\left(f^{-1} \circ o(x)\right)+(1-r) o(x)$, there is a neighbourhood $U$ of 0 in $\mathbb{R}^{n}$ such that

$$
\left\|P g_{r}(x)\right\| \geq\|P(o(x))\|-\|P(f(o(x))-o(x))\| \geq \frac{1}{2}\|o(x)\|>0
$$

for all $x \in U \backslash\{0\}$ and all $r \in[0,1]$.

It follows from Lemma 4.1 that $g_{r}$ is a seed for $(\pi, 0)$ for all $r \in[0,1]$ which induces an orientation $\left\langle g_{r}, \pi\right\rangle$ by Proposition 4.5. In view of Lemma 3.8, $\left\langle g_{r}, \pi\right\rangle$ does not depend on $r$.

Moreover, since $g_{r}$ is a seed for $(\pi, 0), f \circ g_{r}$ is a seed for $(f[\pi], 0)$. We thus have $\langle o, f[\pi]\rangle=\left\langle f \circ g_{1}, f[\pi]\right\rangle=\left\langle f \circ g_{0}, f[\pi]\right\rangle=\langle f \circ o, f[\pi]\rangle$. 
We need to show that

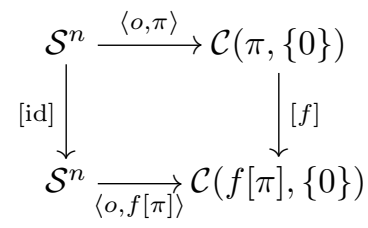

is commutative.

Let $\left(N_{1}, N_{2}\right)$ be a strongly $\pi$-admissible FM-index pair for $(\pi,\{0\})$. Then there are $\lambda \in] 0,1]$ and $s \in \mathbb{R}^{+}$such that

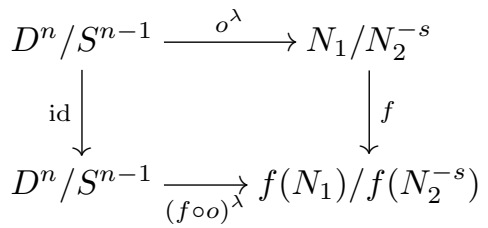

is commutative in $\mathcal{T O P}$. Since $o$ is a seed for $f[\pi]$, we can assume without loss of generality (choosing $\lambda$ and $s$ large enough) that

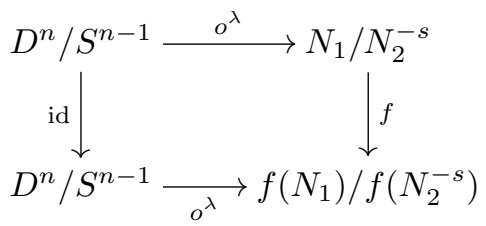

is defined. It commutes because $\langle f \circ o, f[\pi]\rangle=\langle o, f[\pi]\rangle$ as we have already seen.

Since $f$ induces an isomorphism $N_{1} / N_{2}^{-s} \rightarrow f\left(N_{1}\right) / f\left(N_{2}^{-s}\right)$ in $\mathcal{H} \mathcal{T}$, it follows that $\langle o, f[\pi]\rangle=\langle f \circ o, f[\pi]\rangle$ is an orientation.

\section{Orientations for linear skew product semiflows}

For the convenience of the reader, we give a short overview of [6], on which the second part of this paper relies. We begin with an abstract setting for the proof of Theorem 1.2. Subsequently, we introduce a class of linear skew product semiflows, which are crucial for the calculation of the connecting homomorphisms.

Let $H$ be a real Hilbert space, and let $A_{H}: D\left(A_{H}\right) \subset H \rightarrow H$ be a sectorial operator such that:

(1) $A_{H}$ has compact resolvent;

(2) $A_{H}$ is densely defined;

(3) $\Re \lambda>0$ for all $\lambda \in \sigma\left(A_{H}\right)$.

Let $X$ be a real Banach space with continuous inclusion $X \subset H$, and let

$$
A: D(A) \subset X \rightarrow X
$$


be a sectorial operator such that:

(1) $A$ is densely defined;

(2) $A$ has compact resolvent;

(3) $A x=A_{H} x$ for all $x \in D(A)$.

Fix $\alpha \in\left[0,1\left[\right.\right.$, let $X^{\alpha}$ denote the $\alpha$-th fractional power space [5], and let $f \in C^{1}\left(\mathcal{U}, X^{0}\right)$, where $\mathcal{U} \subset X^{\alpha}$ is open.

We consider mild solutions of the Cauchy problem

$$
\dot{x}+A x=f(x),
$$

which induce a local semiflow on $X^{\alpha}$ ([5, Theorem 3.3.3], [1, Theorem A.3]). This semiflow is denoted by $\pi_{f}$, respectively $\pi$ whenever the meaning is clear.

Now, let $e^{-}, e^{+}$be hyperbolic equilibria of (6.1), and assume that the spectrum of $A-D f\left(e^{ \pm}\right)$consists of simple and real eigenvalues. Furthermore, let $u: \mathbb{R} \rightarrow X^{\alpha}$ be a solution of $\pi$ with $u(t) \rightarrow e^{+}$as $t \rightarrow \infty$, and suppose that $e^{\lambda t}\left\|u(t)-e^{+}\right\|_{\alpha} \nrightarrow \rightarrow 0$ as $t \rightarrow \infty$ for some $\lambda \in \mathbb{R}^{+}$. Then by [6, Theorem 3.2], one has $\left\|u(t)-e^{+}\right\|_{\alpha}^{-1}\left(u(t)-e^{+}\right) \rightarrow \eta$ as $t \rightarrow \infty$, where $\eta$ is an eigenvector of $A-D f\left(e^{+}\right)$.

Let $E$ denote the $A$-invariant complement of $\operatorname{span}\{\eta\}$ in $X$, and for $\beta \in[0,1]$, let $E^{\beta}:=E \cap X^{\beta}$ be equipped with the $X^{\beta}$-norm $\|\cdot\|_{\beta}$.

Using [6, Theorem 4.1], it now follows that there exist a neighbourhood $U$ of $\operatorname{cl} u(\mathbb{R})$ and a diffeomorphism $G: U \rightarrow V \subset \mathbb{R} \times E^{\alpha}$ such that $G[\pi]$ is a semiflow whose solutions are mild solutions of

$$
\begin{aligned}
\dot{x} & =g_{1}(x, y), \\
\dot{y}+\widetilde{A} y & =g_{2}(x, y),
\end{aligned}
$$

that is, if $(u(t), v(t)), t \in\left[0, T\left[\right.\right.$, is a solution of $G[\pi]$, then $\dot{u}(t)=g_{1}(u(t), v(t))$ for all $t$, and $v(t)$ is a mild solution of

$$
\dot{y}+\widetilde{A} y=g_{2}(u(t), y),
$$

where $\widetilde{A}$ denotes the restriction of $A$ to $E^{1}$.

By the choise of $G$, we can further assume that $G\left(e^{ \pm}\right)=( \pm 1,0)$ and $G(u(t))$ in $[-1,1] \times\{0\}$ for all $t \in \mathbb{R}$. The semiflow $G[\pi]$ is defined by the condition that $G \circ \widetilde{u}$ is a solution of $G[\pi]$ if and only if $\widetilde{u}$ is a solution of $\pi$ with $\widetilde{u}(\mathbb{R}) \subset U$.

Next, we introduce a new name: $\pi_{1}:=G[\pi]$. Scaling in $y$ yields a family $\left(\pi_{\lambda}\right)_{\lambda \in] 0,1]}$ of semiflows, where $(u(t), v(t))$ is a solution of $\pi_{\lambda}$ if and only if $(u(t), \lambda v(t))$ is a solution of $\pi_{1}$. One can show [6, Proposition 5.15] that $\pi_{\lambda}$ has a limit $\pi_{0}$, where $(u(t), v(t))$ is a solution of $\pi_{0}$ if and only if $(u(t), 0)$ is a solution of $\pi_{1}$ and $v(t)$ is a mild solution of

$$
\dot{y}+\widetilde{A} y=g_{2}(u(t), y) .
$$


At this stage, we need an additonal assumption, namely that $[-1,1] \times\{0\}$ is an isolated invariant set relative to $\pi_{1}$. There are well-known conditions ensuring this, for instance the transversal intersection of the global stable manifold of $e^{+}$ and the global unstable manifold of $e^{-}$, but the isolation of $[-1,1] \times\{0\}$ is sufficient for our purposes. We can conclude that $\left(\pi_{\lambda},[-1,1] \times\{0\}\right)_{\lambda \in[0,1]}$ is an $\mathcal{S}$-continuous family.

For the rest of this section, we will deal with semiflows like $\pi_{0}$, which are called linear skew product semiflows.

\subsection{Linear skew product semiflows.}

Definition 6.1. Let $F$ be a Banach space and let $a<b$ be real numbers. A linear skew product semiflow (see also [11]) on (]$a, b[, F)$ is a semiflow $\pi=(\xi, \Phi)$ on $] a, b[\times F$, where

$$
(x, y) \pi t=(x \xi t, \Phi(x, t) y) \quad \text { for all }(t, x, y) \in D(\pi) .
$$

Here, $\xi$ is a flow on $] a, b[$ and for every $(x, t) \in D(\xi)$ we have $\Phi(x, t) \in \mathcal{L}(F, F)$. Let $\operatorname{SK}(] a, b[, F)$ denote the set of all linear skew product semiflows on (]$a, b[, F)$ and let $\pi \in \mathrm{SK}:=\operatorname{SK}([a, b], F) \subset \mathrm{SK}(] a, b[, F)$ if there exists an $\varepsilon>0$ and a $\widetilde{\pi} \in \operatorname{SK}(] a-\varepsilon, b+\varepsilon[, F)$ with $(x, y) \pi t=(x, y) \widetilde{\pi} t$ whenever the left side is defined.

Given a decomposition $F=F_{1} \oplus F_{2}$ into closed subspaces and semiflows $\pi_{1}=$ $\left(\xi, \Phi_{1}\right) \in \operatorname{SK}\left([a, b], F_{1}\right), \pi_{2}=\left(\xi, \Phi_{2}\right) \in \operatorname{SK}\left(\xi, \Phi_{2}\right)$, define $\pi_{1} \oplus \pi_{2} \in \operatorname{SK}([a, b], E)$ by $\pi_{1} \oplus \pi_{2}=\left(\xi, \Phi_{1} \oplus \Phi_{2}\right)$, where $\left(\Phi_{1} \oplus \Phi_{2}\right)(t, x)\left(y_{1} \oplus y_{2}\right)=\Phi_{1}(t, x) y_{1} \oplus \Phi_{2}(t, x) y_{2}$.

We consider linear skew product semiflows which are generated by semilinear parabolic equations and are normalized on the zero-section, that is, the semiflow $\pi=\pi(A, F) \in \operatorname{SK}\left([-2,2], X^{\alpha}\right)$ is induced by mild solutions of

$$
\begin{aligned}
\dot{x} & =1-x^{2}, \\
\dot{y}+A y & =F(x) y .
\end{aligned}
$$

Unfortunately, the right-hand side of the above equation is not necessarily locally Lipschitz continuous if one assumes only that $F$ is a continuous family of linear operators. Therefore, the term mild solution is used as follows: $(u(t), v(t))$ is called a mild solution of (6.2) if $u(t)$ is a solution of the first equation, that is, $\dot{u}(t)=1-u(t)^{2}$, and $v(t)$ is a mild solution of $\dot{y}+A y=F(u(t)) y$.

Suppose that

- $X$ is a Banach space;

- $A$ is sectorial linear operator which is densely defined on $X$ and has compact resolvent;

- $X^{\alpha}$ denotes the $\alpha$-th fractional power space (see [5]); 
and

(1) $F:[-2,2] \rightarrow \mathcal{L}\left(X^{\alpha}, X^{0}\right)$ is sufficiently continuous, that is, there are $-2=x_{0} \leq \cdots \leq x_{n}=2 \in[-2,2]$ such that for every interval $\left[x_{i}, x_{i+1}\right]$, $i \in\{0, \ldots, n-1\}$, there is an $\widetilde{F} \in C\left(\left[x_{i}, x_{i+1}\right], \mathcal{L}\left(X^{\alpha}, X^{0}\right)\right)$ such that $F(x)=\widetilde{F}(x)$ for every $x \in] x_{i}, x_{i+1}[$;

(2) $-1,1 \notin\left\{x_{0}, \ldots, x_{n}\right\}$.

The linear skew product semiflow defined by (6.2) depends continuously on the right-hand side $F$.

Proposition 6.2 [6, Corollary 6.4]. Let $F_{n} \rightarrow F_{0} \in L^{\infty}\left([-2,2], \mathcal{L}\left(X^{\alpha}, X\right)\right)$, $n \in \mathbb{N}$, and suppose that $F_{n}, n \in \mathbb{N} \cup\{0\}$, are sufficiently continuous. Then:

(a) $\pi\left(A, F_{n}\right)$ is a semiflow for all $n \in \mathbb{N} \cup\{0\}$;

(b) $\pi\left(A, F_{n}\right) \rightarrow \pi(A, F)$ and

(c) every closed set $N \subset]-2,2\left[\times X^{\alpha}\right.$ which is bounded with respect to $\|\cdot\|_{\mathbb{R} \times X^{0}}$ is strongly $\pi\left(A, F_{n}\right)$-admissible.

We will consider classes $\mathrm{SK}_{i}, i \in\{-1,0,1,2\}$, of linear skew product semiflows. Higher indices indicate stronger restrictions. Let us make the following additional assumptions: $A$ and $(A-F(1))$ are hyperbolic and have simple eigenvalues, all of which are real; let $\pi=\pi(A, F)$, and let $E^{ \pm}(\pi, e):=E^{ \pm}(e):=P_{e}^{ \pm}(0) X$, $e \in\{-1,1\}$ denote the associated subspaces of $X$, where $P_{\pi, e}^{ \pm}(0):=P_{e}^{ \pm}(0):=$ $P^{ \pm}(0)$ is the projection onto the subspaces which belong to the positive respectively negative part of the spectrum of $L:=A-F(e) . E^{ \pm, \alpha}:=E^{ \pm} \cap X^{\alpha}$ denotes the respective subspace of $X^{\alpha}$.

(6.2) implies that there are exactly two equilibria, namely $(-1,0)$ and $(1,0)$, and both are hyperbolic.

Definition 6.3. Let $\mathrm{SK}_{0}:=\mathrm{SK}_{0}(\alpha, X, A) \subset \mathrm{SK}\left([-2,2], X^{\alpha}\right)$ denote the set of linear skew product semiflows with $\pi \in \mathrm{SK}_{0}$ if and only if

(a) $\pi$ is induced by mild solutions of (6.2), which satisfies the assumptions above;

(b) $K:=[-1,1] \times\{0\}$ is an isolated invariant set relative to $\pi$;

(c) $\operatorname{dim} E^{-}(1)=\operatorname{dim} E^{-}(-1)<\infty$.

Definition 6.4. Let $[-1,1] \subset] a, b[\subset[-2,2]$ and let $h:[a, b] \rightarrow[-2,2]$ be a homeomorphism such that $h(-1)=-1, h(1)=1$. Let $\pi \in \mathrm{SK}_{-1}=$ $\mathrm{SK}_{-1}([a, b], \alpha, X, A) \subset \mathrm{SK}\left([a, b], X^{\alpha}\right)$ denote the set of all semiflows $\pi$ for which there exists an $h$ with the properties above and a $\tilde{\pi} \in \mathrm{SK}_{0}$ such that $(h \circ u(t), v(t))$ is a solution of $\tilde{\pi}$ whenever $(u(t), v(t))$ is a solution of $\pi$.

In the sequel, we are interested in signs of connecting homomorphisms and need a condition which guarantees that these signs do not change under perturbations. Therefore, we introduce a relation between linear skew product semiflows: 
Definition 6.5. Let $\pi_{0}, \pi_{1} \in \mathrm{SK}_{0}$. Then $\pi_{0} \sim \pi_{1}$ if and only if there exists a homotopy, that is, an $\mathcal{S}$-continuous family $\left(\pi_{\lambda},[-1,1] \times\{0\}\right)_{\lambda \in[0,1]}$ such that for all $\lambda \in[0,1]$

(a) $\pi_{\lambda} \in \mathrm{SK}_{0}$, and

(b) $E^{-}\left(\pi_{\lambda},-1\right)$ and $E^{-}\left(\pi_{\lambda}, 1\right)$ are constant.

Let $\mathrm{SK}_{1} \subset \mathrm{SK}_{0}$ denote the subset of all semiflows $\pi(A, F)$ where $F$ is locally constant in a neigbourhood of $\{-1,1\}$, that is, there exists a $\delta>0$ such that for all $x \in]-1-\delta,-1+\delta[$ we have $F(x)=F(-1)$ and for all $x \in] 1-\delta, 1+\delta[$ $F(x)=F(1)$.

Lemma 6.6 ([6, Lemma 6.10]). For every $\pi(A, F) \in \mathrm{SK}_{0}$, there is a $\lambda_{0} \in$ $[0,1]$ such that $\pi(A, F) \sim \pi\left(A, F_{\lambda}\right) \in \mathrm{SK}_{1}$ for all $\lambda \in\left[0, \lambda_{0}\right]$, where we set

$$
F_{\lambda}(x):= \begin{cases}F(-1) & \text { if } x \in[-1-\lambda,-1+\lambda], \\ F(1) & \text { if } x \in[1-\lambda, 1+\lambda], \\ F(x) & \text { otherwise. }\end{cases}
$$

Let $\mathrm{SK}_{2} \subset \mathrm{SK}_{1}$ denote the subset of all those semiflows which satisfy the following stronger restriction (compared to the definition of $\mathrm{SK}_{1}$ ): There exists a $\delta>0$ such that $F(x)=F(-1)$ for all $x \in[-2,-1+\delta[$ and $F(x)=F(1)$ for all $x \in] 1-\delta, 2]$.

Lemma 6.7 ([6, Lemma 6.11]). For every $\pi(A, F) \in \mathrm{SK}_{1}$, it holds that $\pi(A, F) \sim \pi(A, \widetilde{F}) \in \mathrm{SK}_{2}$, where we set

$$
\widetilde{F}(x):= \begin{cases}F(-1) & \text { if }-2 \leq x \leq-1, \\ F(x) & \text { if }-1<x<1, \\ F(1) & \text { if } 1 \leq x \leq 2\end{cases}
$$

For $\pi=(\xi, \Phi) \in \mathrm{SK}_{2}$, we can define an invariant subbundle $U$ (the exact terminology can be found in the appendix). After the definition of $U$, we will show that this subbundle determines the sign of connecting homorphisms.

Let $E^{-}:=E^{-}(\pi,-1)$ and define $U(x) \in \mathcal{L}\left(E^{-}, X^{\alpha}\right)$ by

$$
U(x) y:=y, \quad x \in[-2,-1+\delta], y \in E^{-} .
$$

We continue along $[-2,2]$ by following the semiflow, that is,

$$
U(x):=U(-1+\delta) \Phi\left(-1+\delta, t_{x}\right), \quad x \in[-1+\delta, 1-\delta]
$$

where $(-1+\delta) \xi t_{x}=x$ defines $t_{x}$.

$P_{1}^{-}(0) \circ U(1-\delta)$ is a bijection, so, given $y_{0} \in E^{-}(1)$, there is a $w \in E^{-}(-1)$ with $P_{1}^{-}(0) \circ U(1-\delta) w=y_{0}$. Choose a basis $\left\{\eta_{i}: i=1 \ldots \operatorname{dim} E^{-}(1)\right\}$ for $E^{-}(1)$ such that each $\eta_{i}$ is an eigenvector of $L:=A-F(1)$. 
Further, let $\lambda_{i}<0$ denote the real eigenvalue $\lambda_{i}$ which corresponds to $\eta_{i}$, that is, $e^{-L t} \eta_{i}=e^{-\lambda_{i} t} \eta_{i}$. For each $i \in\left\{1, \ldots, \operatorname{dim} E^{-}\right\}$, there is an $\eta_{i}^{+} \in E^{+}(1)$ with $\eta_{i}+\eta_{i}^{+} \in U(1-\delta) E^{-}(-1)$. Let $y_{i} \in E^{-}$be given by $U(1-\delta) y_{i}=\eta_{i}+\eta_{i}^{+}$ and define

$$
U(x) y_{i}:=\eta_{i}+e^{-\left(L-\lambda_{i}\right)\left(t_{x}-t_{1-\delta}\right)} \eta_{i}^{+}, \quad x \in\left[1-\delta, 1\left[, i=1 \ldots \operatorname{dim} E^{-} .\right.\right.
$$

Finally, let

$$
U(x) y:=\lim _{\widetilde{x} \rightarrow 1} U(\widetilde{x}) y, \quad x \in[1,2], y \in E^{-} .
$$

$U$ is $\pi$-invariant $\left({ }^{6}\right)$, so there is a linear skew product semiflow $\pi_{U}=\left(\xi, \Phi_{U}\right) \in$ $\mathrm{SK}\left([-2,2], E^{-}\right)$such that

$$
U(x \xi t) \Phi(x, t) y=\Phi_{U}(x, t) U(x) y
$$

whenever $x \xi[0, t] \subset]-2,2[$.

\subsection{Conley index orientations for linear skew product semiflows.}

Lemma 6.8. Let $\pi=(\xi, \Phi) \in \mathrm{SK}_{-1}([a, b], \alpha, X, A)$ and let $\Psi_{-1}, \Psi_{1} \in$ $\mathcal{L}\left(\mathbb{R}^{n}, X^{\alpha}\right)$, such that $P_{-1}^{-}(\pi, 0) \Psi_{-1}$ and $P_{1}^{-}(\pi, 0) \Psi_{1}$ are isomorphisms. Then:

(a) $\left.o_{-1}(x, y):=\left(-1, \Psi_{-1} y\right),(x, y) \in\right]-1 / 2,1 / 2\left[\times \mathbb{R}^{n}\right.$, is a seed for $(\pi$, $(-1,0))$, and

(b) $o_{1}(y):=\left(1, \Psi_{1} y\right), y \in \mathbb{R}^{n}$, is a seed for $(\pi,(1,0))$.

If $\pi \in \mathrm{SK}_{1}$, then the semiflow is linear in the sense of Section 2 in a neighbourhood of each of the equilibria $( \pm 1,0)$. In this case, Proposition 2.3 implies that $\pi$ is strongly linearizable (Definition 2.4) in each of the equilibria, so we can invoke Proposition 4.5, which states that $o_{ \pm 1}$ are seeds.

However, the notion of being strongly linearizable relies on the existence of some kind of tangential space for stable sets. We have not established such a result for $\mathrm{SK}_{0}$, so we will circumvent this problem by using the more elementary Lemma 3.4 and the concrete structure of our linear skew product semiflows.

Proof. Suppose that $\pi=\pi(A, F)$.

(a) Let $U:=B_{1 / 2}[(-1,0)] \subset[-2,2] \times X^{\alpha}$, and let $\left(N_{1}, N_{2}\right)$ be a strongly $\pi$-admissible FM-index pair for $(\pi,(-1,0))$ with $N_{1} \subset U$. Since $\operatorname{Inv}^{+}(U) \subset$ $\{0\} \times E^{+, \alpha}(-1)$, it follows that $o_{-1}(x, y) \in N_{2}^{-\infty}$ for all $(x, y) \in D\left(o_{-1}\right) \backslash\{0\}$. Now, Lemma 3.4 implies that $o_{-1}$ is a seed for $(\pi,(-1,0))$.

(b) Let $X_{1}:=\{0\} \times X^{\alpha}$ and $\left(N_{1}, N_{2}\right)$ be a strongly $\pi$-admissible FM-index pair for $(\pi,(1,0))$ with $N_{1} \subset B_{1}[(1,0)] \subset \mathbb{R} \times X^{\alpha}$. Then $\left(X_{1} \cap N_{1}, X_{1} \cap N_{2}\right)$

$\left({ }^{6}\right)$ Here, we identify $U$ with its image. 
is a strongly $\pi$-admissible FM-index pair for $(\tilde{\pi}, 0)$, where $\tilde{\pi}$ is induced by mild solutions of the linear equation

$$
\begin{aligned}
\dot{x}, & =0 & & x \in\{0\}, \\
\dot{y}+A y & =F(1) y, & & y \in X^{\alpha} .
\end{aligned}
$$

It follows from Corollary 4.6 that $o_{1}$ is a seed for $(\tilde{\pi}, 0)$, that is, there is a $\lambda \in \mathbb{R}^{+}$ such that $o_{1}^{\lambda}(y)=o(\lambda y) \in N_{2}^{-\infty}$ for all $y \in \mathbb{R}^{n} \backslash\{0\}$. As before, applying Lemma 3.4 proves that $o_{1}$ is a seed for $(\pi,(-1,0))$.

Until further notice, let $\mu$ be given by Definition 5.1, $\pi \in \mathrm{SK}_{-1}, \Psi_{-1} \in$ $\operatorname{ISO}\left(\mathbb{R}^{n}, E^{-}(-1)\right), \Psi_{1} \in \operatorname{ISO}\left(\mathbb{R}^{n}, E^{-}(1)\right)$, and $o_{1}$ and $o_{-1}$ be defined by Lemma 6.8 .

Definition 6.9.

$$
\bar{\theta}(\pi):=\bar{\theta}\left(\pi, \mu, \Psi_{-1}, \Psi_{1}\right):=\theta\left(H_{n-1}\left\langle o_{1}\right\rangle^{-1} \circ \partial_{n} \circ H_{n}\left\langle o_{-1}\right\rangle, \mu, n, 1\right)
$$

where $\partial_{q}: H_{q}\langle\pi,\{(-1,0)\}\rangle \rightarrow\langle\pi,\{(1,0)\}\rangle$ denotes the $q$-th connecting homorphism of the long exact attractor-repeller sequence in singular homology which is associated with $(\pi,[-1,1] \times\{0\},\{(1,0)\},\{(-1,0)\})$.

LEMMA 6.10. Let $\pi_{k} \rightarrow \pi_{\infty}$ be a sequence in $\mathrm{SK}_{0}$ such that the assumptions of $[2$, Theorem 7.3] hold whenever $\widetilde{N}$ is a bounded neighbourhood of $[-1,1] \times\{0\}$. Suppose that $\left\langle o_{-1}, \pi_{k},(-1,0)\right\rangle$ (resp. $\left.\left\langle o_{1}, \pi_{k},(1,0)\right\rangle\right)$ is an orientation for all $k \in$ $\mathbb{N} \cup\{\infty\}$ sufficiently large. Then $\bar{\theta}\left(\pi_{k}\right)=\bar{\theta}\left(\pi_{\infty}\right)$ for all $k \in \mathbb{N}$ sufficiently large.

Proof. By [2, Theorem 7.3], there are strongly admissible FM-index triples $\left(N_{1, k}, N_{2, k}, N_{3, k}\right),\left(\widetilde{N}_{1, k}, \widetilde{N}_{2, k}, \widetilde{N}_{3, k}\right)$ for $\left(\pi_{k},[-1,1] \times\{0\},\{(1,0)\},\{(-1,0\})\right.$ and $\left(M_{1}, M_{2}, M_{3}\right),\left(\widetilde{M}_{1}, \widetilde{M}_{2}, \widetilde{M}_{3}\right)$ for $\left(\pi_{\infty},[-1,1] \times\{0\},\{(1,0)\},\{(-1,0)\}\right)$ such that for all $k \in \mathbb{N}$ sufficiently large

$$
\left(\widetilde{N}_{1, k}, \widetilde{N}_{2, k}, \widetilde{N}_{3, k}\right) \subset\left(\widetilde{M}_{1}, \widetilde{M}_{2}, \widetilde{M}_{3}\right) \subset\left(N_{1, k}, N_{2, k}, N_{3, k}\right) \subset\left(M_{1}, M_{2}, M_{3}\right)
$$

We can assume that $M_{1}$ is bounded in $X$ so that it is strongly $\pi_{\infty}$-admissible by Proposition 6.2.

It follows from Proposition 3.10 that

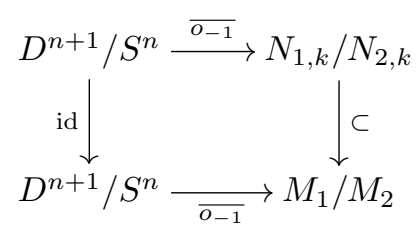


and

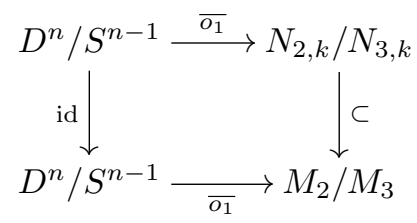

are commutative for all $k \in \mathbb{N}$ sufficiently large.

Moreover, there is a commutative ladder

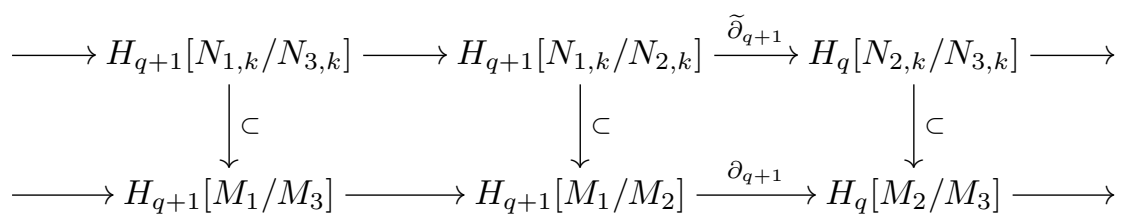

where $\partial_{q}$ and $\widetilde{\partial}_{q}$ denote the respective $q$-th connecting homomorphism.

It follows that $\bar{\theta}\left(\pi_{k}\right)=\bar{\theta}\left(\pi_{\infty}\right)$ for all $k$ sufficiently large.

Proposition 6.11. $\left\langle o_{\nu}, \pi,(\nu, 0)\right\rangle$ is an orientation for every $\pi \in \mathrm{SK}_{0}, \nu \in$ $\{-1,1\}$. Moreover, for all $\pi_{0}, \pi_{1} \in \mathrm{SK}_{0}$, it holds that $\bar{\theta}\left(\pi_{0}\right)=\bar{\theta}\left(\pi_{1}\right)$ whenever $\pi_{0} \sim \pi_{1}$ in the sense of Definiton 6.5.

Proof. First, assume that $\pi \in \mathrm{SK}_{1}$, let $\nu \in\{-1,1\}, m=n$ for $\nu=1$ and $m=n+1$ for $\nu=-1$. Then there is a neighbourhood $U$ of $(\nu, 0)$ in $]-2,2\left[\times X^{\alpha}\right.$ such that the restriction of $\pi$ to $U$ is induced by mild solutions of

$$
\begin{aligned}
\dot{x} & =1-x^{2}, \\
\dot{y}+A y & =F(\nu) y .
\end{aligned}
$$

It follows from Corollary 4.6 that $\left\langle o_{\nu}, \pi\right\rangle$ is an orientation.

Now, let $\pi \in \mathrm{SK}_{0}$. By Lemma 6.6, there is an $\mathcal{S}$-continous family $\left(\pi_{\lambda},\{(\nu, 0)\}\right)$ such that $\pi_{1} \in \mathrm{SK}_{1}, \pi_{0}=\pi$, and $E^{-}\left(\pi_{\lambda}, \nu\right)$ are constant. Hence, $o_{\nu}$ is a seed for $\left(\pi_{\lambda},(\nu, 0)\right)$ for every $\lambda \in[0,1]$. It follows from Theorem 3.12 that $\left\langle o_{\nu}, \pi\right\rangle$ is an orientation for $(\pi,\{\nu\})$, proving the first claim.

In order to show the second claim, let $\pi_{0}, \pi_{1} \in \mathrm{SK}_{0}$ with $\pi_{0} \sim \pi_{1}$, that is, there exists an $\mathcal{S}$-continuous family $\left(\pi_{\lambda},[-1,1] \times\{0\}\right)_{\lambda \in[0,1]}$ such that $E^{-}\left(\pi_{\lambda},-1\right)$ and $E^{-}\left(\pi_{\lambda}, 1\right)$ are constant. Therefore, we can choose $\Psi_{1}$ and $\Psi_{-1}$ such that $o_{-1}$ (resp. $\left.o_{1}\right)$ induces orientations for $\left(\pi_{\lambda},-1\right)$ (resp. $\left.\left(\pi_{\lambda}, 1\right)\right)$ for all $\lambda \in[0,1]$.

Suppose that $\bar{\theta}\left(\pi_{\lambda}\right)$ is not constant. Then there is a sequence $\lambda_{n} \rightarrow \lambda_{\infty}$ in $[0,1]$ with $\bar{\theta}\left(\pi_{n}\right) \neq \bar{\theta}\left(\pi_{\infty}\right)$, where we set $\pi_{n}:=\pi_{\lambda_{n}}, n \in \mathbb{N} \cup\{\infty\}$. This is a contradiction to Lemma 6.10 , showing that $\bar{\theta}\left(\pi_{0}\right)=\bar{\theta}\left(\pi_{1}\right)$. 
6.3. The unstable subbundle. For every $\pi \in \mathrm{SK}_{2}$, we have defined an invariant subbundle $U$ of $[-2,2] \times X^{\alpha}$. Let $\left(N_{1}, N_{2}, N_{3}\right)$ be an arbitrary FMindex pair for $(\pi,[-1,1] \times\{0\},\{(1,0)\},\{(-1,0)\})$. Due to the invariance of $U$, $\left(M_{1}, M_{2}, M_{3}\right):=\left(N_{1} \cap U, N_{2} \cap U, N_{3} \cap U\right)$ is an FM-index pair for $\pi_{\mid \operatorname{im} U}$ (recall that we have already defined a semiflow $\pi_{U}$, which denotes the restriction of $\pi$ to $U$.

The inclusion $\left(M_{1}, M_{2}, M_{3}\right) \subset\left(N_{1}, N_{2}, N_{3}\right)$ induces a commutative ladder in singular homology, namely

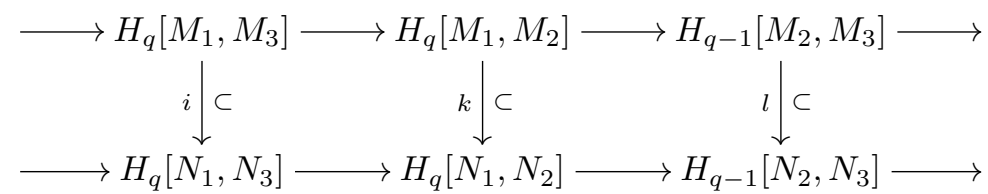

Lemma 6.12. For every $\pi \in \mathrm{SK}_{2},\left\langle o_{\nu}, \pi_{\mid \operatorname{im} U}\right\rangle, \nu \in\{-1,1\}$, induces an orientation for $\left(\pi_{\mid \operatorname{im} U},\{(\nu, 0)\}\right)$.

Proof. $F(x)$ is constant for all $x$ in a neighbourhood $N_{ \pm 1}$ of \pm 1 . Therefore, $U( \pm 1)=E^{-}( \pm 1)$, and so $o_{-1}$ and $o_{1}$ can be defined by Lemma 6.8. It follows from Corollary 4.6 that $\left\langle o_{\nu}, \pi,(\nu, 0)\right\rangle$ is an orientation for every $\nu \in\{-1,1\}$.

Lemma 6.12 guarantees that $\bar{\theta}\left(\pi_{\mid \mathrm{im} U}\right)$ is defined and so we may formulate the following:

Proposition 6.13. For every $\pi \in \mathrm{SK}_{2}$ it holds that $\bar{\theta}(\pi)=\bar{\theta}\left(\pi_{\mid \operatorname{im} U}\right)$.

Proof. Let $\nu \in\{-1,1\}$ and $\left(N_{1}, N_{2}\right)$ be an arbitrary strongly admissible FM-index pair for $(\pi,\{(\nu, 0)\})$. Then $\left(N_{1} \cap U, N_{2} \cap U\right)$ is a strongly admissible FM-index pair for $\left(\pi_{\mid \operatorname{im} U},\{(\nu, 0)\}\right)$.

By Lemma 6.12, there is an $s \in \mathbb{R}^{+}$and a $\lambda \in[0,1]$ such that $o_{\nu}^{\lambda}\left(S^{m-1}\right) \subset$ $N_{2}^{-s} \cap U \subset N_{2}^{-s}$, where $m=n$ for $\nu=1$ and $m=n+1$ for $\nu=-1$. Therefore,

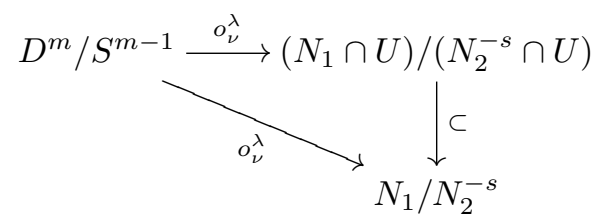

is commutative in $\mathcal{T O P}$ and thus in $\mathcal{H} \mathcal{T}$,

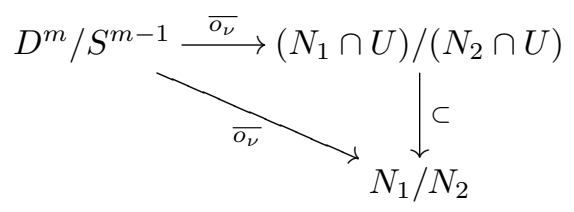


Now, let $\left(N_{1}, N_{2}, N_{3}\right)$ be a strongly admissible FM-index triple for $(\pi,[-1,1]$ $\times\{0\},\{(1,0)\},\{(-1,0)\})$. It follows that $H_{q}(i)$ and $H_{q}(l)$ (defined in (6.5)) are isomorphisms since $\overline{o_{\nu}}$ is an isomorphism by Proposition 6.11 respectively Lemma 6.12. Therefore, the commutativity of (6.5) implies that $\bar{\theta}\left(\pi_{\mid \operatorname{im} U}\right)=\bar{\theta}(\pi)$ as claimed.

The definition $\operatorname{sgn}_{\Psi_{-1}, \Psi_{1}} U:=\operatorname{sgn} \operatorname{det} \Psi_{1}^{-1} U(1) U(-1)^{-1} \Psi_{-1}$ gives $U$ a sign. We define the inverse of an injective and continuous homorphism $A \in \mathcal{L}(E, F)$ on $\operatorname{im} A \subset F$. The definition of $\operatorname{sgn} U$ makes sense because

$$
\operatorname{im} \Psi_{-1}=E^{-}(-1)=\operatorname{im} U(-1) \quad \text { and } \quad \operatorname{im} \Psi_{1}=E^{-}(1)=\operatorname{im} U(1) .
$$

Alternatively, one can read the inverses in the above equation as left inverses. In this case, $\operatorname{sgn}_{\Psi_{-1}, \Psi_{1}} U$ is well-defined and agrees with the first definition.

Recall that the definition of $\theta$ requires a choice of generators $\mu=\left(\mu_{q}\right)_{q \in \mathbb{Z}}$. Consider the following system

$$
\begin{aligned}
& \dot{x}=1-x^{2}, \\
& \dot{y}=y,
\end{aligned}
$$

of ordinary differential equations on $]-2,2\left[\times \mathbb{R}^{n}\right.$. They define a semiflow $\chi_{n}$, which is obviously a linear skew product semiflow, that is, $\chi_{n} \in \mathrm{SK}_{2}\left([-2,2], \mathbb{R}^{n}\right)$. Let $U_{\chi_{n}}$ denote the subbundle $U$ which is defined with respect to $\chi_{n}$ (in fact, $\left.U_{\chi_{n}}=[-2,2] \times \mathbb{R}^{n}\right)$.

Definition 6.14. Let $\widetilde{\mu}_{0}: \mathbb{Z} \rightarrow H_{0}\left(\mathcal{S}^{0}\right)$ be arbitrary, and let $\mu=\left(\mu_{q}\right)_{q \in \mathbb{Z}}$ be such that $\mu_{0}=\widetilde{\mu}_{0}$ and for all $n \in \mathbb{N}$

$$
\bar{\theta}\left(\chi_{n}, \mu, \mathrm{id}_{\mathbb{R}^{n}}, \mathrm{id}_{\mathbb{R}^{n}}\right)=\operatorname{sgn}_{\mathrm{id}_{\mathbb{R}^{n}}, \mathrm{id}_{\mathbb{R}^{n}}} U_{\chi_{n}}
$$

It is clear that $\mu$ is well-defined, and the following proposition shows that the definition makes sense.

Proposition 6.15. For every $\pi \in \mathrm{SK}_{2}$, for every $\Psi_{-1} \in \operatorname{ISO}\left(\mathbb{R}^{n}, E^{-}(-1)\right)$, and for every $\Psi_{1} \in \operatorname{ISO}\left(\mathbb{R}^{n}, E^{-}(1)\right)$, it holds that

$$
\bar{\theta}\left(\pi,\left(\mu_{q}\right)_{q \in \mathbb{Z}}, \Psi_{-1}, \Psi_{1}\right)=\operatorname{sgn}_{\Psi_{-1}, \Psi_{1}} U_{\pi} \neq 0 .
$$

Proof. By [6, Proposition 6.23], there is an isomorphism of trivial bundles $V:=V_{\pi_{U}}$ such that $\tilde{\chi}:=V^{-1}\left[\pi_{U}\right] \sim \chi_{n}$.

As usual, let $o_{1}$ and $o_{-1}$ be given by Lemma 6.8. They are seeds for an orientation for $(-1,0)$ respectively $(1,0)$. Moreover, $\widehat{o}_{-1}:=(-1,0)+\mathrm{id}_{\mathbb{R}^{n}}$ is a seed for $(\widetilde{\chi},(-1,0))$ and $\widehat{o}_{1}:=(1,0)+\operatorname{id}_{\mathbb{R}^{n}}$ is a seed for $(\widetilde{\chi},(1,0))$. 
Let $\alpha$ be defined by

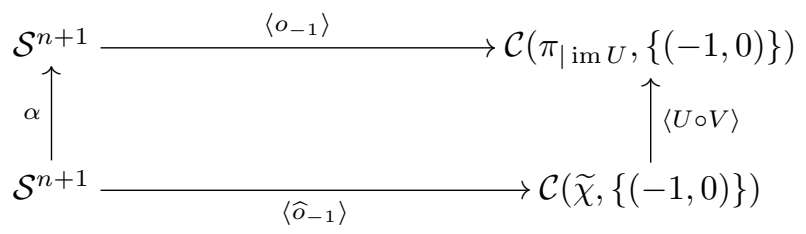

and $\beta$ by

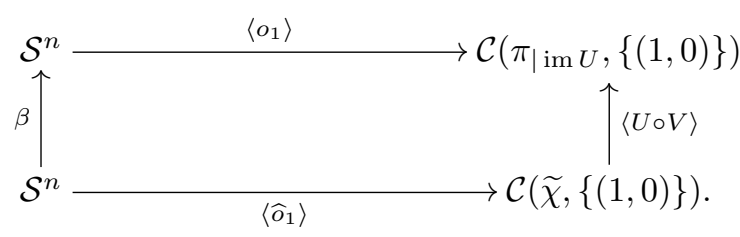

It follows from Proposition 4.7 and Corollary 4.6 that

$$
\alpha=\operatorname{sgn} \operatorname{det}\left(\begin{array}{cc}
1 & 0 \\
0 & \Psi_{-1}^{-1} \circ U(-1) \circ V(-1)
\end{array}\right)=\operatorname{sgn} \operatorname{det} \Psi_{-1}^{-1} \circ U(-1) \circ V(-1)
$$

and

$$
\beta=\operatorname{sgn} \operatorname{det} \Psi_{1}^{-1} \circ U(1) \circ V(1) .
$$

Since $\operatorname{sgn} \operatorname{det} V(1) \circ V(-1)=\operatorname{sgn} \operatorname{det} V(1) \circ V(1)$ (by homotopy, $V$ is a continuous family of isomorphisms), it follows that $\beta \circ \alpha^{-1}=\operatorname{sgn}_{\Psi_{-1}, \Psi_{1}} U$, where we denote the mappings by their mapping degree.

In singular homology, the respective attractor-repeller sequences define a commutative diagram:

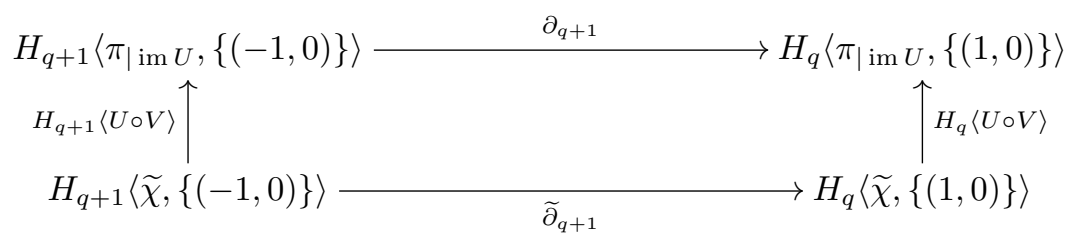

It follows from Proposition 6.11 and the choice of $\mu$ that $\bar{\theta}\left(\tilde{\chi}, \mu, \mathrm{id}_{\mathbb{R}^{n}}, \mathrm{id}_{\mathbb{R}^{n}}\right)=$ $\bar{\theta}\left(\chi_{n}, \mu, \operatorname{id}_{\mathbb{R}^{n}}, \operatorname{id}_{\mathbb{R}^{n}}\right)=1$. We obtain a commutative diagram

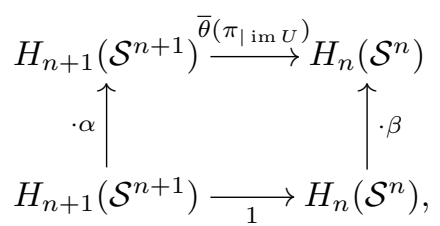

showing that $\bar{\theta}\left(\pi_{\mid \operatorname{im} U}\right)=\alpha \beta=\operatorname{sgn}_{\Psi_{-1}, \Psi_{1}} U$. 
6.4. Geometric orientation. Let $\Psi_{-1} \in \operatorname{ISO}\left(\mathbb{R}^{n}, E^{-}(-1)\right)$ and $\Psi_{1} \in \mathcal{L} \in$ $\operatorname{ISO}\left(\mathbb{R}^{n}, E^{-}(1)\right)$ be arbitrary but fixed as in the previous section. We will define a geometric orientation for every $\pi \in \mathrm{SK}_{2}$ and then show that this geometric orientation is well-defined for every $\pi \in \mathrm{SK}_{0}$ and coincides with the (Conley index) orientation of the previous section.

Definition 6.16. For every $\pi=(\xi, \Phi) \in \mathrm{SK}_{-1}$, let $\operatorname{sgn} \pi \in\{-1,1\}$ denote the unique number for which

$$
\operatorname{sgn} \pi:=\operatorname{sgn}\left(\pi, \Psi_{-1}, \Psi_{1}\right):=\lim _{(x, x \xi t) \rightarrow(-1+, 1-)} \operatorname{sgn} \operatorname{det} \Psi_{1}^{-1} P \Phi(x, t) \Psi_{-1},
$$

where $P=P_{1}^{-}(0)$ denotes the unique projection $P: X \rightarrow E^{-}(1)$ with $\operatorname{ker} P=$ $E^{+}(1)$.

Note that for every $t \in \mathbb{R}^{+}$, the spaces $E^{-}(1)$ and $E^{+, \alpha}(1)$ (resp. $E^{-}(-1)$ and $\left.E^{+, \alpha}(1)\right)$ are $\Phi(1, t)$-invariant (resp. $\Phi(-1, t)$-invariant) subspaces.

Lemma 6.17. Let $\pi=\pi(A, F) \in \mathrm{SK}_{1}$ and $\delta>0$ such that

$$
\begin{array}{ll}
F(x)=F(-1), & x \in[-1,-1+\delta], \\
F(x)=F(1), & x \in[1-\delta, 1] .
\end{array}
$$

Then

$$
\operatorname{sgn} \pi=\operatorname{sgn} \operatorname{det} \Psi_{1}^{-1} P \Phi\left(-1+\delta, t_{0}\right) \Psi_{-1} \neq 0,
$$

where $(-1+\delta) \xi t_{0}=1-\delta$. In particular, $\operatorname{sgn} \pi$ is well-defined for every $\pi \in \mathrm{SK}_{1}$.

Proof. Let $x \in]-1,1\left[\right.$ and $t \in \mathbb{R}^{+}$such that $\left.\left.x \in\right]-1,-1+\delta\right]$ and $x \xi t \geq 1-\delta$. Then there are $t_{-1}, t_{1} \in \mathbb{R}^{+}$such that $x \xi t_{1}=-1+\delta$, and $(1-\delta) \xi t_{1}=x \xi t$. We have

$$
\begin{aligned}
P \Phi(x, t) & =P \Phi\left(1-\delta, t_{1}\right) \Phi\left(-1+\delta, t_{0}\right) \Phi\left(x, t_{-1}\right) \\
& =P \Phi\left(1-\delta, t_{1}\right) P \Phi\left(-1+\delta, t_{0}\right) \Phi\left(x, t_{-1}\right) .
\end{aligned}
$$

$P \Phi\left(1-\delta, t_{1}\right) \Phi\left(-1+\delta, t_{0}\right) \Phi\left((-1+\delta) \xi\left(-\lambda t_{-1}\right), \lambda t_{-1}\right) \Psi_{-1}$ is an ismorphism for all $\lambda \in[0,1]$. Otherwise, there would be a $0 \neq \widetilde{y} \in E^{-}(-1)$, an $\left.\left.\widetilde{x} \in\right]-1,-1+\delta\right]$, and a $\widetilde{t} \in \mathbb{R}^{+}$with $\widetilde{x} \xi \widetilde{t} \geq 1-\delta$ and $\Phi(\widetilde{x}, \widetilde{t}) \widetilde{y} \in E^{+}(1)$. This implies that there exists a full bounded solution through $(\widetilde{x}, \widetilde{y})$, which contradicts the isolation of $[-1,1] \times\{0\}$ relative to $\pi$ (see [6, Lemma 6.8]).

We have shown that

$$
\operatorname{sgn} \operatorname{det} \Psi_{1}^{-1} P \Phi(x, t) \Psi_{-1}=\operatorname{sgn} \operatorname{det} P \Phi\left(1-\delta, t_{1}\right) P \quad P \Phi\left(-1+\delta, t_{0}\right) \Psi_{-1} .
$$

A similar argument applies to $P \Phi(1-\delta, s) P$. It is an isomorphism for all $s \in\left[0, t_{1}\right]$ and homotopic to the identity on $\left(E^{-}(1), E^{-}(1) \backslash\{0\}\right)$, showing that

$$
\operatorname{sgn} \operatorname{det} \Psi_{1}^{-1} P \Phi(x, t) \Psi_{-1}=\operatorname{sgn} \operatorname{det} \Psi_{1}^{-1} P \Phi\left(-1+\delta, t_{0}\right) \Psi_{1} .
$$


The following proposition relies on Proposition 6.15 .

Proposition 6.18. Let $\pi \in \mathrm{SK}_{1}$. Then $\operatorname{sgn} \pi=\bar{\theta}(\pi) \neq 0$.

Proof. Recall that for every $\pi \in \mathrm{SK}_{1}$ there is a $\delta=\delta(\pi)>0$ such that

$$
\begin{array}{ll}
F(x)=F(-1), & x \in[-1,-1+\delta], \\
F(x)=F(1), & x \in[1-\delta, 1],
\end{array}
$$

and we have $U(x)=E^{-}(-1)$ for all $x \in[-2,-1+\delta]$.

Initially, suppose that $\pi \in \mathrm{SK}_{2}$. Let $\left.x \in\right]-1,-1+\delta\left[, t \in \mathbb{R}^{+}\right.$, and $\pi_{U}=$ $\left(\xi, \Phi_{U}\right)$. We have

$$
\Phi(x, t) y=U(x \xi t) \Phi_{U}(x, t) U(x)^{-1} y
$$

for all $y \in E^{-}(-1)$.

Moreover, we have sgn $\operatorname{det} \Phi_{U}\left(x_{0}, t_{0}\right)=1$ since $\Phi_{U}(x, t) \in \operatorname{ISO}\left(E^{-}(-1)\right.$, $\left.E^{-}(-1)\right)$ for all $(x, t) \in D\left(\Phi_{U}\right)$. It follows from (6.6) that

$$
\operatorname{sgn} \operatorname{det} \Psi_{1}^{-1} P \Phi(x, t) \Psi_{-1}=\operatorname{sgn} \operatorname{det} \Psi_{1}^{-1} P U(x \xi t) U(x)^{-1} \Psi_{-1} .
$$

Taking $(6.7)$ to the limit $(x, x \xi t) \rightarrow(-1,1)$, we obtain $\operatorname{sgn} \pi=\operatorname{sgn} U$, proving in conjunction with Proposition 6.15 that $\operatorname{sgn} \pi=\operatorname{sgn} U=\bar{\theta}(\pi)$.

Lemma 6.6 states that for every $\pi_{0} \in \mathrm{SK}_{1}$ there is a $\pi_{1} \in \mathrm{SK}_{2}$ with $\pi_{0} \sim \pi_{1}$. It follows immediately from the differential equation given there that $\operatorname{sgn} \pi_{0}=$ $\operatorname{sgn} \pi_{1}$. Moreover, Proposition 6.11 implies that $\bar{\theta}\left(\pi_{0}\right)=\bar{\theta}\left(\pi_{1}\right)$. This proves the claim for every $\pi \in \mathrm{SK}_{1}$.

LEMMA 6.19. Let $\Psi_{\nu, k}, \nu \in\{-1,1\}$, be a sequence of homomorphisms in $\mathcal{L}\left(\mathbb{R}^{n}, X^{1}\right)$ with $\Psi_{\nu, k} \rightarrow \Psi_{\nu, \infty} \in \operatorname{ISO}\left(\mathbb{R}^{n}, E^{-}(\nu)\right)$ as $k \rightarrow \infty$. Then, for every $\pi=(\xi, \Phi) \in \mathrm{SK}_{0}$, one has

$$
\begin{aligned}
\lim _{(x, x \xi t, k) \rightarrow(-1+, 1, \infty)} \operatorname{sgn} \operatorname{det}\left(P \Psi_{1, k}\right)^{-1} P \Phi(x, t) \Psi_{-1, k} & \\
& =\bar{\theta}\left(\pi, \Psi_{-1, \infty}, \Psi_{1, \infty}\right) \neq 0 .
\end{aligned}
$$

Proof. Let $x_{k} \rightarrow-1$ in $[-1,1], t_{k} \in \mathbb{R}^{+}$with $x_{k} \xi t_{k} \rightarrow 1, \pi_{0}=\pi(A, F) \in$ $\mathrm{SK}_{0}$, and $\pi_{k}:=\pi\left(A, F_{k}\right)$ with

$$
F_{k}(x):= \begin{cases}F(-1) & \text { if }-2+x_{k} \leq x<x_{k}, \\ F(1) & \text { if } x_{k} \xi t_{k} \leq x<2-\left(x_{k} \xi t_{k}\right), \\ F(x) & \text { otherwise. }\end{cases}
$$

We have $F_{k} \rightarrow F$ as $k \rightarrow \infty$ in $L^{\infty}\left([-2,2], \mathcal{L}\left(X^{\alpha}, X\right)\right)$. Moreover, there is a strongly admissible isolating neighbourhood for $[-1,1] \times\{0\}$ relative to $\pi$, so we can choose $k_{0} \in \mathbb{N}$ such that $[-1,1] \times\{0\}$ is an isolated invariant set relative 
to $\pi_{k}$ for all $k \geq k_{0}$. Consequently, one has $\pi_{k} \in \mathrm{SK}_{1}$ for all $k_{0} \leq k<\infty$. We can assume without loss of generality that $P \Psi_{1, k}$ is an isomorphism for $k \geq k_{0}$.

If $(u(t), v(t)), t \in[0, T]$, is a solution of $\pi_{0}$ with $x_{k} \leq u(t) \leq x_{k} \xi t_{k}$ for all $t \in[0, T]$, then it is also a solution of $\pi_{k}$. Hence, it follows from Lemma 6.17 and Proposition 6.18 that for all $k_{0} \leq k<\infty$

$$
\operatorname{sgn} \operatorname{det}\left(P \Psi_{1, k}\right)^{-1} P \Phi\left(x_{k}, t_{k}\right) \Psi_{-1, k}=\bar{\theta}\left(\pi_{k}, \Psi_{-1, k}, P \Psi_{1, k}\right) .
$$

As shown in the proof of Proposition 6.11, every $\widetilde{\pi} \in \mathrm{SK}_{1}$ is strongly linearizable in the sense of Definition 2.2 in each of its equilibria. Thus, it follows from Corollary 4.6 and Proposition 4.7 that there is a $k_{1} \geq k_{0}$ such that

$$
\bar{\theta}\left(\pi_{k}, \Psi_{-1, k}, P \Psi_{1, k}\right)=\bar{\theta}\left(\pi_{k}, \Psi_{-1, \infty}, \Psi_{1, \infty}\right) \quad \text { for all } k_{1} \leq k<\infty .
$$

Finally, in view of Lemma 6.10 , there is a $k_{2} \geq k_{1}$ such that

$$
\bar{\theta}\left(\pi_{k}, \Psi_{-1, \infty}, \Psi_{1, \infty}\right)=\bar{\theta}\left(\pi, \Psi_{-1, \infty}, \Psi_{1, \infty}\right) \quad \text { for all } k_{2} \leq k \leq \infty .
$$

An immediate consequence of Lemma 6.19 is

COROllary 6.20. $\operatorname{sgn} \pi$ is well-defined for every $\pi \in \mathrm{SK}_{0}$ and we have $\bar{\theta}(\pi)=\operatorname{sgn}(\pi)$.

Corollary 6.21. Let $\pi=(\xi, \Phi) \in \mathrm{SK}_{-1}([a, b], \alpha, X, A)$. Then $\operatorname{sgn} \pi$ is well-defined and we have $\operatorname{sgn} \pi=\bar{\theta}(\pi)$. Moreover, Lemma 6.19 holds for every $\pi \in \mathrm{SK}_{-1}$.

Proof. According to Definition 6.4, there is a semiflow $\widetilde{\pi}=(\widetilde{\xi}, \Phi) \in \mathrm{SK}_{0}$ such that $(h(u(t)), v(t))$ is a solution of $\widetilde{\pi}$ whenever $(u(t), v(t))$ is a solution of $\pi$.

This shows immediately that $\operatorname{sgn} \pi$ is well-defined and $\operatorname{sgn} \pi=\operatorname{sgn} \tilde{\pi}$. It is also clear that

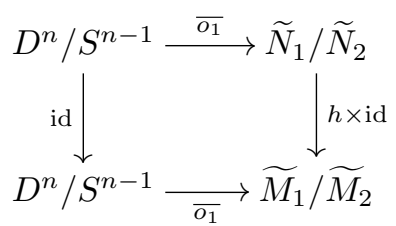

is commutative whenever $\left(\widetilde{N}_{1}, \widetilde{N}_{2}\right)$ is a strongly $\pi$-admissible FM-index pair for $(\pi,\{(1,0)\})$ and $\left(\widetilde{M}_{1}, \widetilde{M}_{2}\right)=(h \times$ id $)\left(\widetilde{N}_{1}, \widetilde{N}_{2}\right)$.

Since $h$ is necessarily strictly monotone increasing,

$$
g_{\lambda}(x):=\lambda(h \times \mathrm{id}) \circ o_{-1}(x)+(1-\lambda) o_{-1}(x)
$$

satisfies $g_{\lambda}(x) \neq(-1,0)$ for all $x \in D^{n} \backslash\{0\}$. Given an arbitrary $\lambda \in[0,1]$, it is a straightforward extension of Lemma 6.8 that $g_{\lambda}$ is a seed for $(\pi,\{(-1,0)\})$ 
and $(\widetilde{\pi},\{(-1,0)\})$. Hence, by Lemma 3.8,

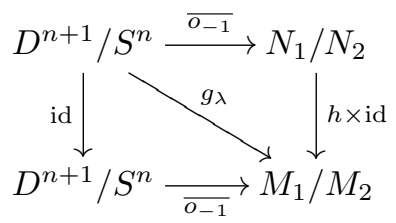

commutes in $\mathcal{H} \mathcal{T}$ for all $\lambda \in[0,1]$, where $\left(N_{1}, N_{2}\right)$ is a strongly admissible FMindex pair for $(\pi,\{(-1,0)\})$ and $\left(M_{1}, M_{2}\right)=(h \times$ id $)\left(N_{1}, N_{2}\right)$.

Therefore, we have $\bar{\theta}(\pi)=\bar{\theta}(\widetilde{\pi})$. The left-hand side of (6.8) is unaffected by $h$, showing that the formula still holds.

\section{Heteroclinic solutions}

Recall the assumptions of the beginning of the the previous section. In particular let $u: \mathbb{R} \rightarrow X^{\alpha}$ be a solution of (6.1) with $u(t) \rightarrow e^{ \pm}$as $t \rightarrow \pm \infty$. It follows that $\left\|u(t)-e^{+}\right\|_{\alpha}^{-1}\left(u(t)-e^{+}\right) \rightarrow \eta \in X^{1}$ as $t \rightarrow \infty$. $\eta$ is an eigenvector of $A-D f\left(e^{+}\right)$which belongs to an eigenvalue $\lambda>0$.

Let $E \subset X$ be an $A$-invariant and $A-D f\left(e^{+}\right)$invariant subspace $\left({ }^{7}\right)$ with $X=E \oplus\{\eta\}$. By $E=E_{1} \oplus E_{2}$, we mean that $E_{1}$ and $E_{2}$ are closed linear subspaces of $E$ with $E_{1} \cap E_{2}=\{0\}$ and $E=E_{1}+E_{2}$. The canonical projection $P: E_{1} \oplus E_{2} \rightarrow E_{1}$ is given by $P\left(e_{1} \oplus e_{2}\right):=e_{1}$.

Due to the hyperbolicity of $A-D f\left(e^{+}\right)$, there is a decomposition $E=$ $E^{-}(-1) \oplus E^{+}(-1)$, where $E^{-}(-1)\left(\right.$ resp. $\left.E^{+}(-1) \cap X^{1}\right)$ is a $A-D f\left(e^{+}\right)$invariant subspace and the restriction $\widetilde{A}^{-}$of $A-D f\left(e^{+}\right)$to $E^{-}(-1)$ (resp. $\widetilde{A}^{+}$of $A-$ $D f\left(e^{+}\right)$to $\left.E^{+}(-1)\right)$ satisfies $\Re \sigma\left(\widetilde{A}^{-}\right)<0$ (resp. $\Re \sigma\left(\widetilde{A}^{+}\right)>0$ ).

In view of [6, Theorem 4.1], we can assume that:

(1) $G\left(e^{+}\right)=(1,0), G\left(e^{-}\right)=(-1,0)$;

(2) $G(u(t)) \in]-1,1[\times\{0\}$ for all $t \in \mathbb{R}$;

(3) $D G(x) y=(0, y)$ for all $y \in E$ and for all $x$ in a neighbourhood of $e^{+}$.

Let $\pi_{1}:=G\left[\pi_{1}\right]$, and let the family of semiflows $\left(\pi_{\lambda}\right)_{\lambda \in[0,1]}$ be defined by scaling in $y$ as explained in the the previous section. It follows from [6, Theorem 5.12] that $\left(\pi_{\lambda},[-1,1] \times\{0\}\right)_{\lambda \in[0,1]}$ is $\mathcal{S}$-continuous. Note that $\pi_{0} \in \mathrm{SK}_{-1}$.

Definition 7.1. Let $\left\{x_{1}, \ldots, x_{n+1}\right\}$ be a basis for $E_{X}^{-}\left(e^{-}\right)$consisting of eigenvectors of $A-D f\left(e^{-}\right)$, let $\left\{y_{1}, \ldots, y_{n}\right\}$ be a basis for $E_{X}^{-}\left(e^{+}\right)$, and let $\Psi_{-1}:=\left(x_{1}, \ldots, x_{n+1}\right)$ and $\Psi_{1}:=\left(y_{1}, \ldots, y_{n}\right)$ denote the corresponding matrices, which we understand as isomorphisms $\mathbb{R}^{n+1} \rightarrow E_{X}^{-}\left(e^{-}\right)\left(\right.$resp. $\mathbb{R}^{n} \rightarrow E_{X}^{-}\left(e^{+}\right)$).

Let $P(t)$ denote the canonical projection

$$
P(t): E^{-}(+1) \oplus \operatorname{span}\{\dot{u}(t)\} \oplus E^{+}(+1) \rightarrow E^{-}(+1) .
$$

$\left({ }^{7}\right)$ This can always be achieved by choosing $A$ appropriately. 
$P(t)$ is well defined for large $t \in \mathbb{R}$. Define

$$
\begin{aligned}
& \nu(u):=\nu\left(u, \Psi_{-1}\right):=(-1)^{i+1} \operatorname{sgn} \widetilde{\nu}, \\
& \widehat{\Psi}=\left(x_{1}, \ldots, x_{i-1}, x_{i+1}, \ldots, x_{n+1}\right),
\end{aligned}
$$

and

$$
\operatorname{sgn} u:=\nu(u) \cdot \lim _{(t, t+\Delta) \rightarrow(-\infty, \infty)} \operatorname{sgn} \operatorname{det} \Psi_{1}^{-1} P(t+\Delta) D \Pi_{\Delta}(u(t)) \widehat{\Psi}
$$

where $\left(u(-t)-e^{-}\right)\left\|u(-t)-e^{-}\right\|_{\alpha}^{-1} \rightarrow \widetilde{\nu} x_{i}\left\|x_{i}\right\|_{\alpha}^{-1}$ as $t \rightarrow \infty$ and $\Pi_{t} x:=x \pi t$.

It is clear that $\operatorname{sgn} u$ depends on the isomorphisms $\Psi_{-1}$ and $\Psi_{1}$, that is, $\operatorname{sgn} u=\operatorname{sgn}\left(u, \Psi_{-1}, \Psi_{1}\right)$.

$\bar{u}=\operatorname{cl}\{u(t): t \in \mathbb{R}\}$ is an isolated invariant set, and $\left(\bar{u},\left\{e^{+}\right\},\left\{e^{-}\right\}\right)$is an attractor-repeller decomposition $\left(\left\{e^{+}\right\}\right.$denotes the attractor). There is a long exact sequence in singular homology associated with the attractor-repeller decomposition. Let $\left(\partial_{q}\right)_{q \in \mathbb{Z}}$ denote the family of connecting homomorphisms of this sequence, that is, $\partial_{q+1}: H_{q+1}\left\langle\pi,\left\{e_{-1}\right\}\right\rangle \rightarrow H_{q}\left\langle\pi,\left\{e_{1}\right\}\right\rangle$ for all $q \in \mathbb{Z}$.

Definition 7.2. Let $\theta$ be given by Definition 5.1, $\mu$ by Definition 6.14, and let

$$
\widehat{\theta}(\pi, u):=\widehat{\theta}\left(\pi, u, \Psi_{-1}, \Psi_{1}\right):=\theta\left(H_{n}\left\langle\widehat{o}_{1}\right\rangle \circ \partial_{n+1} \circ H_{n+1}\left\langle\widehat{o}_{-1}\right\rangle, \mu, n+1,1\right),
$$

where we set

$$
\begin{array}{ll}
\widehat{o}_{-1}(y):=e^{-}+\Psi_{-1}(y), & y \in \mathbb{R}^{n+1}, \\
\widehat{o}_{+1}(y):=e^{+}+\Psi_{+1}(y), & y \in \mathbb{R}^{n} .
\end{array}
$$

It follows from Proposition 2.3 that $\pi$ is strongly linearizable at $e^{+}$and $e^{-}$, so Proposition 4.5 implies that $\widehat{o}_{i}, i \in\{-1,1\}$, induces an orientation. Thus, $\widehat{\theta}$ is defined.

Let $p_{1}: \mathbb{R} \times E \rightarrow \mathbb{R}\left(\right.$ resp. $\left.p_{2}: \mathbb{R} \times E \rightarrow E\right), p_{1}(x, y):=x\left(\right.$ resp. $\left.p_{2}(x, y):=y\right)$, denote the projection onto the first (resp. second) component.

Proposition 7.3. $\widehat{\theta}\left(\pi, u, \Psi_{-1}, \Psi_{1}\right)=\nu\left(u, \Psi_{-1}\right) \cdot \bar{\theta}\left(\pi_{0}, \widetilde{\Psi}_{-1}, \widetilde{\Psi}_{1}\right)$, where we set

$$
\widetilde{\Psi}_{-1}:=p_{2} \circ D G\left(e^{-}\right) \circ \widehat{\Psi},
$$

and

$$
\widetilde{\Psi}_{1}:=p_{2} \circ D G\left(e^{+}\right) \circ \Psi_{1} .
$$

Note that our assumptions at the beginning of this section imply that $\left(0, \widetilde{\Psi}_{1} y\right)=\left(D G\left(e^{+}\right) \circ \Psi_{1}\right) y$ for all $y \in \mathbb{R}^{n}$. 
Proof. Define

$$
\begin{aligned}
o_{-1}(x, y) & :=\left(-1+x, \widetilde{\Psi}_{1}(y)\right), & & (x, y) \in \mathbb{R} \times \mathbb{R}^{n}, \\
o_{1}(y) & :=\left(1, \widetilde{\Psi}_{1}(y)\right), & & y \in \mathbb{R}^{n},
\end{aligned}
$$

as in Lemma 6.8 and consider the following commutative diagram

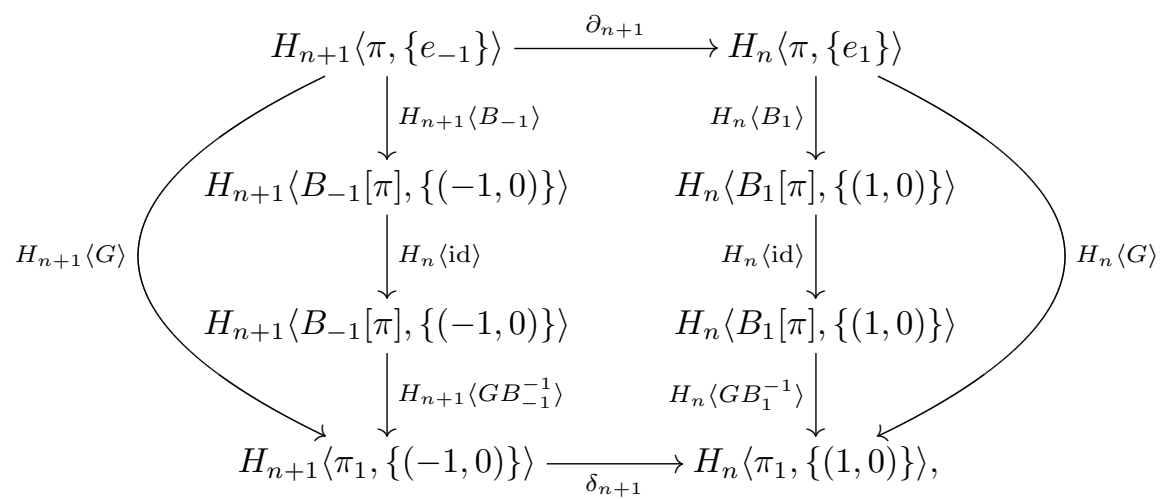

where we set

$$
B_{-1}\left(e^{-}+x\right):=(-1,0)+D G\left(e^{-}\right) x, \quad B_{1}\left(e^{+}+x\right):=(1,0)+D G\left(e^{+}\right) x .
$$

$\delta_{q}: H_{q}\left\langle\pi_{1},\{(-1,0)\}\right\rangle \rightarrow H_{q-1}\left\langle\pi_{1},\{(1,0)\}\right\rangle$ is the connecting homomorphism associated with $\left(\pi_{1},[-1,1] \times\{0\},\{(1,0)\},\{(-1,0)\}\right)$.

Applying orientations, we obtain for $i \in\{-1,1\}$

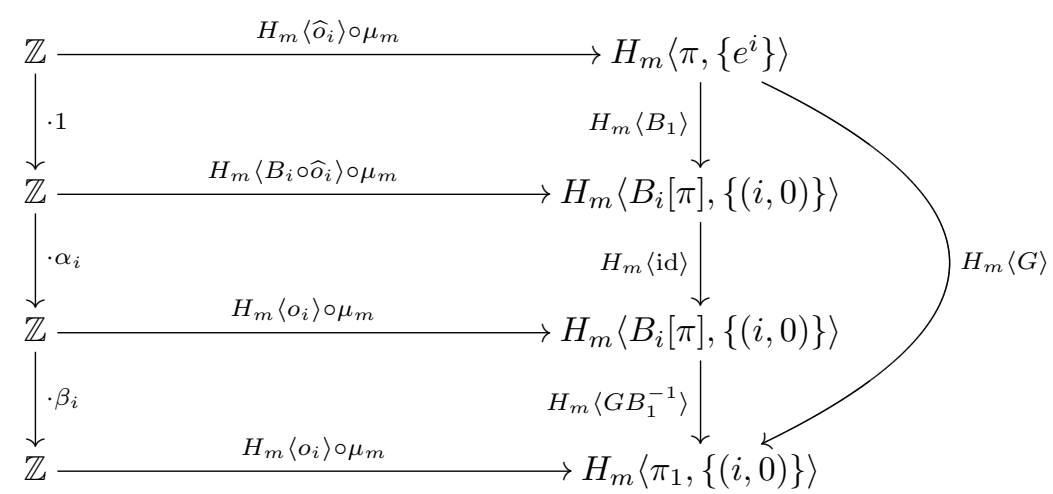

where we set

$$
m:= \begin{cases}n+1 & \text { if } i=-1 \\ n & \text { if } i=1 .\end{cases}
$$

It follows from Proposition 5.3 that $\beta_{-1}=\beta_{1}=1$. We thus have (relative to these orientations)

$$
\widehat{\theta}\left(\pi, u, \widehat{o}_{-1}, \widehat{o}_{1}\right)=\alpha_{1} \alpha_{-1} \widetilde{\theta}\left(\delta_{n+1}\right),
$$

where we set $\tilde{\theta}(\delta):=\theta\left(H_{n}\left\langle o_{1}\right\rangle^{-1} \circ \delta \circ H_{n+1}\left\langle o_{-1}\right\rangle, \nu, n+1,1\right)$. 
One has $\alpha_{1}=1$ because $B_{1} \circ \widehat{o}_{1}=o_{1}$. By Proposition 4.7 we further have

$$
\alpha_{-1}=\operatorname{sgn} \operatorname{det}\left(\Psi_{1}^{-1} \circ D G\left(e_{-1}\right)^{-1} \circ\left(1, \widetilde{\Psi}_{-1}\right)\right),
$$

where $\left(1, \widetilde{\Psi}_{-1}\right)\left(y_{1}, y_{2}\right)=\left(y_{1}, \widetilde{\Psi}_{-1} y_{2}\right)$.

Since $\left(u(t)-e^{-}\right)\left\|u(t)-e^{-}\right\|_{\alpha}^{-1} \rightarrow \widetilde{\nu} x_{i}\left\|x_{i}\right\|_{\alpha}^{-1}$ in $X^{\alpha}$ as $t \rightarrow-\infty$, one has $D G\left(e^{-}\right)\left(\widetilde{\nu} x_{i}\left\|x_{i}\right\|_{\alpha}^{-1}\right)=(1,0)$, so written as matrices $\left(^{8}\right)$

$$
\Psi_{-1}^{-1} D G\left(e^{-}\right)^{-1}\left((1,0), \widetilde{\Psi}_{-1}\right) \sim\left(\widetilde{\nu} \widetilde{e}_{i}, \widetilde{e}_{1}, \ldots, \widetilde{e}_{i-1}, \widetilde{e}_{i+1}, \ldots \widetilde{e}_{n+1}\right) .
$$

Here, $\left((1,0), \widetilde{\Psi}_{-1}\right)\left(x_{1}, \ldots, x_{n+1}\right):=x_{1} \cdot(1,0)+\widetilde{\Psi}_{-1}\left(x_{2}, \ldots, x_{n+1}\right), \widetilde{e}_{k}:=\Psi_{-1} x_{k} k$ denotes the $k$-th unity vector in $\mathbb{R}^{n+1}$, and given $C, D \in \operatorname{ISO}\left(\mathbb{R}^{n+1}\right.$, $\mathbb{R}^{n+1}$ ), we write $C \sim D$ if and only if $\operatorname{det} C \operatorname{det} D>0$. This shows that $\alpha_{-1}=(-1)^{i+1} \widetilde{\nu}=\nu(u)$.

It follows from $\left[6\right.$, Proposition 5.15] that $\left(\pi_{\lambda},[-1,1] \times\{0\}\right)_{\lambda \in[0,1]}$ is $\mathcal{S}$-continuous and for every $\lambda \in[0,1],([-1,1],\{1\},\{-1\})$ is an attractor-repeller decomposition relative to $\pi_{\lambda}$. Let $\delta_{n+1}^{\lambda}: H_{n+1}\left\langle\pi_{\lambda},\{(-1,0)\}\right\rangle \rightarrow H_{n}\left\langle\pi_{\lambda},\{(1,0)\}\right\rangle$ denote the associated connecting homomorphism in singular homology.

We will show that $\lambda \mapsto \widetilde{\theta}\left(\delta_{n+1}^{\lambda}\right)=: \widetilde{\theta}_{\lambda}$ is locally constant. Otherwise, there is a sequence $\lambda_{k} \rightarrow \lambda_{0}$ in $[0,1]$ such that $\theta_{k}:=\widetilde{\theta}\left(\delta^{\lambda_{k}}\right) \neq \widetilde{\theta}\left(\delta^{\lambda_{0}}\right)=: \theta_{0}$. It follows from [2, Theorem 7.3] that for all $k$ large enough, there are strongly admissible FM-index triples $\left(N_{1, k}, N_{2, k}, N_{3, k}\right)$ and $\left(\tilde{N}_{1, k}, \widetilde{N}_{2, k}, \widetilde{N}_{3, k}\right)$ for $\pi_{k}:=\pi_{\lambda_{k}}, k \in$ $\mathbb{N} \cup\{0\}$ such that the following diagram (the rows of which are a part of the respective long exact attractor repeller sequence in homology)

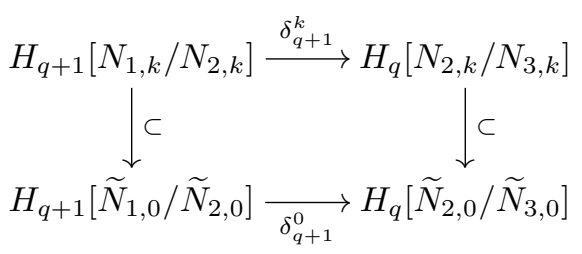

is defined, commutative, and its vertical arrows denote isomorphisms $\left({ }^{9}\right)$.

Now, Proposition 3.10 implies that $\theta_{k}=\theta_{0}$ for all $k \in \mathbb{N}$ sufficiently large, a contradiction, and so $\bar{\theta}\left(\pi_{0}, \widetilde{\Psi}_{-1}, \widetilde{\Psi}_{1}\right)=\theta_{0}=\theta_{1}=\widetilde{\theta}\left(\delta_{n+1}\right)$.

TheOREM 7.4. $\operatorname{sgn} u:=\operatorname{sgn}\left(u, \Psi_{-1}, \Psi_{1}\right)$ is well-defined and

$$
\partial_{q+1} \circ H_{q+1}\left\langle\widehat{o}_{-1}\right\rangle \circ \mu_{q+1}= \begin{cases}\operatorname{sgn} u \cdot H_{q}\left\langle\widehat{o}_{1}\right\rangle \circ \mu_{q} & \text { if } q=n, \\ 0 & \text { otherwise. }\end{cases}
$$

$\left.{ }^{8}\right)\left(y_{1}, \ldots, y_{n}\right)\left(x_{1}, \ldots, x_{n}\right):=x_{1} \cdot y_{1}+\cdots+x_{n} \cdot y_{n}$ for $\left(x_{1}, \ldots, x_{n}\right) \in \mathbb{R}^{n}$.

$\left({ }^{9}\right)$ The respective inclusion induced morphism in the homotopy category of pointed spaces is a homotopy equivalence and therefore induces an isomorphism in singular homology. 
Note that the seeds $\widehat{o}_{ \pm 1}$ and the sign of $u$ depend on $\Psi_{ \pm 1}$. The assumptions on $G$ at the beginning of this section are used throughout the following proof without further notice.

Proof. Let $v(t):=p_{1} \circ G \circ u(t)$. Lemma 5.16 in [6] relates the semigroup $\Pi_{t}$ to the linear skew product semiflow $\pi_{0}=(\xi, \Phi)$, namely

$$
p_{2} D \widetilde{\Pi}_{\Delta}(v(t))=\Phi(v(t), \Delta) p_{2},
$$

where we set $\widetilde{\Pi}_{t} x:=G(x \xi t) \Pi_{t} G(x)^{-1}=x \pi_{1} t$. Recall that Definition 6.16 relies on the canonical projection

$$
P: E^{-}(-1) \oplus E^{+}(-1) \rightarrow E^{-}(-1) .
$$

Let $P(t)$ be given by Definition 7.1. Translating to $\mathbb{R} \times E$, we obtain

$$
\widetilde{P}(t):=D G(u(t)) P(t) D G(u(t))^{-1} .
$$

We have

$$
P(t) D G(u(t))^{-1}(x, y)=P(t) \widetilde{x} \dot{u}(t)+P y
$$

for some $\widetilde{x} \in \mathbb{R}$, so we can drop the notation of $t$ that is, $\widetilde{P}:=\widetilde{P}(t)$, where $t$ is large (so that $P(t)$ is defined) but, apart from that, arbitrary.

Defining

$$
\begin{array}{rlrl}
\widetilde{\Psi}_{1, t} & :=D G(u(t)) \Psi_{1}, & \widetilde{\Psi}_{1}:=D G\left(e^{+}\right) \Psi_{1}, \\
\widetilde{\Psi}_{-1, t}:=D G(u(t)) \widehat{\Psi}, & \widetilde{\Psi}_{-1}:=D G\left(e^{-}\right) \widehat{\Psi},
\end{array}
$$

we further have $\widetilde{\Psi}_{1, t}=\widetilde{\Psi}_{1}$ for all $t \in \mathbb{R}$ with $|t|$ sufficiently large, and $\widetilde{\Psi}_{-1, t} \rightarrow$ $\widetilde{\Psi}_{-1}$ as $t \rightarrow-\infty$.

It follows from Corollary 6.21 that

$$
\operatorname{sgn} \operatorname{det}\left(p_{2} \widetilde{\Psi}_{1}\right)^{-1} P \Phi(v(t), \Delta) p_{2} \widetilde{\Psi}_{-1, t} \rightarrow \bar{\theta}\left(\pi_{0}, \widetilde{\Psi}_{-1}, \widetilde{\Psi}_{1}\right) \neq 0
$$

as $(t, t+\Delta) \rightarrow(-\infty, \infty)$.

For fixed parameters $t$ and $\Delta$, one has

$$
P \Phi(v(t), \Delta) p_{2}=\widetilde{P} D \widetilde{\Pi}_{\Delta}(v(t)),
$$

so it follows from (7.2) that

$$
\operatorname{sgn} \operatorname{det} \widetilde{\Psi}_{1}^{-1} \widetilde{P} D \widetilde{\Pi}_{\Delta}(v(t)) \widetilde{\Psi}_{-1, t} \rightarrow \bar{\theta}\left(\pi_{0}, \widetilde{\Psi}_{-1}, \widetilde{\Psi}_{1}\right) \neq 0 .
$$

We have

$$
\widetilde{\Psi}_{1}^{-1} \widetilde{P} D \widetilde{\Pi}_{\Delta}(v(t)) \widetilde{\Psi}_{-1, t}=\Psi_{1}^{-1} P(t) D \Pi_{\Delta}(v(t)) \widehat{\Psi},
$$

showing that $\operatorname{sgn}\left(u, \Psi_{-1}, \Psi_{1}\right)$ is defined. Using Proposition 7.3, one obtains

$$
\operatorname{sgn}\left(u, \Psi_{-1}, \Psi_{1}\right)=\widehat{\theta}\left(\pi, u, \Psi_{-1}, \Psi_{1}\right) .
$$

Resolving the definition of $\widehat{\theta}$ completesthe proof. 


\section{Appendix}

Although one could certainly use the notion of a vector bundle as defined in [7], this would create a large overhead due to formalism since the structure of the vector bundles used here is relatively simple.

Let $[a, b] \subset \mathbb{R}$ be fixed and let $E, F$ denote arbitrary Banach spaces. We will write $E=E_{1} \oplus E_{2}$ if and only if $E_{1}$ and $E_{2}$ are closed linear subspaces of $E$ with $E=E_{1}+E_{2}$ and $E_{1} \cap E_{2}=\{0\}$. Given a linear subspace $E_{1} \subset E$, another linear subspace $E_{2}$ is called a topological complement if and only if $E=E_{1} \oplus E_{2}$. In particular, such a complement exists if $E_{1}$ is closed and either $\operatorname{dim} E_{1}<\infty$ or $\operatorname{codim} E_{1}<\infty$.

Definition 8.1. A (trivial) bundle is the Cartesian product $[a, b] \times E$ equipped with the product metric.

Taking (trivial) bundles as objects of a category $\mathcal{B}=\mathcal{B}([a, b])$, one needs to define morphisms:

Definition 8.2. A morphism in $\mathcal{B}$ is a continuous mapping $G:[a, b] \rightarrow$ $\mathcal{L}(E, F) . \quad G$ is called a splitting if for every $x \in[a, b], G(x) E$ has a topological complement in $F$.

Given bundles $[a, b] \times E$ and $[a, b] \times \widetilde{E}$ and a morphism $F$ between them, $F$ can be applied to $[a, b] \times E$ in the following way: $\widehat{F}(x, \eta):=(x, F(x) \eta)$.

If $F_{1}, F_{2}$ are morphisms, then $\left(F_{1} \circ F_{2}\right)(x):=F_{1}(x) \circ F_{2}(x)$ is again a morphism. In particular, a morphism $F$ is an isomorphism iff for every $x \in[a, b]$ $F(x) \in \mathcal{L}(E, F)$ is an isomorphism and iff the induced mapping $\widehat{F}$ is a homeomorphism.

Lemma 8.3. Let $G \in C([a, b], \mathcal{L}(E, F))$ and suppose that $G\left(x_{0}\right)$ is an isomorphism in $\mathcal{L}(E, F)$. Then there is a neighbourhood $U$ of $x_{0}$ in $[a, b]$ such that $G(x)$ is an isomorphism for all $x \in U$. Moreover, $G(x)^{-1}$ is continuous in $x$ for all $x \in U$.

Corollary 8.4. $G \in C([a, b], \mathcal{L}(E, F))$ is an isomorphism if and only if for every $x \in[a, b] G(x)$ is an isomorphism in $\mathcal{L}(E, F)$.

Definition 8.5. A subset $U \subset[a, b] \times F$ is called a subbundle if there exists another bundle $[a, b] \times E$ and a splitting monomorphism $G:[a, b] \times E \rightarrow[a, b] \times F$ such that $U=\widehat{G}([a, b] \times E)$.

LEMMA 8.6. $\widehat{G}:[a, b] \times E \rightarrow U$ is a homeomorphism, and the norms on the fibers are equivalent, that is, there are constants $m, M \in \mathbb{R}^{+}$such that $0 \neq m$ and $m\|\eta\|_{E} \leq\|G(x) \eta\|_{F} \leq M\|\eta\|_{E}$ for all $(x, \eta) \in[a, b] \times E$.

Given a splitting monomorphism $U:[a, b] \times E \rightarrow[a, b] \times F$, one can speak of a subbundle, identifying $U$ with its image $\widehat{U}([a, b] \times E)$. Then the fibers are 
given by $U(x):=U(x) E$ for $x \in[a, b]$. If $V \subset[a, b]$, then we write $U(V):=$ $\bigcup_{x \in V}\{x\} \times U(x)$.

LEMma 8.7. Let $U:[a, b] \times E \rightarrow[a, b] \times F$ be a subbundle, let $x_{0} \in[a, b]$ and let $P: F \rightarrow U\left(x_{0}\right)$ be a continuous projection onto $U\left(x_{0}\right)$. Then there exists a neighbourhood $V$ of $x_{0}$ in $[a, b]$ such that $p: U(V) \rightarrow V \times U\left(x_{0}\right), p(x, y)=(x, P y)$, is a homeomorphism and the norms on the fibers are equivalent, that is, there are constants $m, M \in \mathbb{R}^{+}$such that $0 \neq m$ and $m\|\eta\| \leq\|P \eta\| \leq M\|\eta\|$ for all $(x, \eta) \in U(V)$.

\section{REFERENCES}

[1] N. ACKermann And T. BARtsch, Superstable manifolds of semilinear parabolic problems, J. Dynam. Differential Equations 17 (2005), 115-173.

[2] M.C. Carbinatto and K.P. Rybakowski, Homology index braids in infinite-dimensional Conley index theory, Topol. Methods Nonlinear Anal. 26 (2005), 35-74.

[3] A. Floer, Witten's complex and infinite-dimensional Morse theory, J. Differential Geometry 30 (1989), 207-221.

[4] A. Hatcher, Algebraic Topology, Cambridge University Press, 2002.

[5] D. Henry, Geometric theory of semilinear parabolic equations, Lecture Notes in Mathematics, vol. 840, Springer-Verlag, 1981.

[6] A. JäNIG, The Conley index along heteroclinic solutions of reaction-diffusion equations, J. Differential Equations 252 (2012), 4410-4454.

[7] S. LAng, Fundamentals of Differential Geometry, Graduate Texts in Mathematics, vol. 191, Springer, New York, 1999.

[8] J. M. LeE, Introduction to Smooth Manifolds, Graduate Texts in Mathematics, vol. 218, Springer, 2003.

[9] K.P. Rybakowski, The Homotopy Index and Partial Differential Equations, Springer, 1987.

[10] D. Salamon, Morse theory, the Conley index and Floer homology, Bull. London Math. Soc. 22 (1990), 113-140.

[11] G.R. Sell and Y. You, Dynamics of Evolutionary Equations, Applied Mathematical Sciences, vol. 143, Springer, New York, 2002.

[12] T. Tom Dieck, Topologie, de Gruyter, 1991.

AXEL JÄNIG

Universität Rostock

Institut für Mathematik

18051 Rostock, GERMANY

E-mail address: axel.jaenig@uni-rostock.de 